\title{
Tracing bulk elemental ratios in exoplanetary atmospheres with TiO chemistry
}

\author{
Vanesa Ramírez ${ }^{1}$, Alexander J. Cridland ${ }^{1}$, and Paul Mollière ${ }^{2}$ \\ ${ }^{1}$ Leiden Observatory, Leiden University, 2300 RA Leiden, The Netherlands \\ e-mail: cridland@strw. leidenuniv.nl \\ 2 Max-Planck-Institut für Astronomie, Königstuhl 17, 69117 Heidelberg, Germany
}

Received 17 April 2020 / Accepted 1 July 2020

\begin{abstract}
Deciphering the bulk elemental abundances of exoplanetary atmospheres is not an easy task, yet it is crucial to understanding the formation history of planets. The purpose of this work is to show that the observability of TiO features at optical wavelengths in the transmission spectra of hot Jupiter atmospheres is sensitive to the bulk chemical properties of the atmosphere. To this end, we ran a grid of chemical models, which include TiO formation and destruction, for the ultra-hot Jupiter WASP-19b and an ultra-hot version of HD 209458b. We take into account non-equilibrium chemistry and changes in the temperature and pressure structure of these atmospheres caused by different $\mathrm{C} / \mathrm{O}$ ratios. We calculated synthetic transmission spectra for these models, and studied the relative strengths of $\mathrm{TiO}$ and $\mathrm{H}_{2} \mathrm{O}$ features quantitatively. To compare with observations, we used a model-independent metric for molecular abundances, $\Delta Z_{\mathrm{TiO}_{-} \mathrm{H}_{2} \mathrm{O}} / H_{\mathrm{eq}}$, which has previously been used in observational studies of exoplanetary atmospheres. We find that with this metric we can differentiate between different chemical models and place constraints on the bulk carbon and oxygen abundances of the atmosphere. From chemical considerations, we expected the $\mathrm{TiO}$ abundance to depend on the bulk nitrogen. However, we find that changes in $\mathrm{N} / \mathrm{H}$ do not result in changes in the resulting TiO. We applied our method to a set of known exoplanets that have been observed in the relevant optical wavelengths and find good agreement between low-resolution observations and our model for WASP-121b, marginally good agreement with WASP-79b, WASP-76b, and WASP-19b, and poorer agreement with HD 209458b. Our method will be particularly helpful for indirect studies of the bulk abundances of carbon and oxygen.
\end{abstract}

Key words. planets and satellites: atmospheres - astrochemistry - planets and satellites: composition planets and satellites: gaseous planets

\section{Introduction}

The bulk chemical properties of an exoplanetary atmosphere its carbon-to-oxygen $(\mathrm{C} / \mathrm{O})$ and nitrogen-to-oxygen $(\mathrm{N} / \mathrm{O})$ ratios - are impacted by the location and timing of the gas accretion phase of its formation (Öberg et al. 2011; Helling et al. 2014; Madhusudhan et al. 2014; Cridland et al. 2016, 2019a; Pudritz et al. 2018; Oberg et al. 2019, among many others). These ratios depend on the chemical structure of the gas and ice throughout the protoplanetary disc (Öberg et al. 2011; Eistrup et al. 2018), on the chemical properties of the refractory material (i.e. dust and planetesimals, Mordasini et al. 2016; Cridland et al. 2019b) that is accreted directly into the atmosphere, and on the migration history of the growing planet (Madhusudhan et al. 2014; Cridland et al. 2017).

Characterising the elemental ratios of exoplanetary atmospheres observationally is crucial to probing the details of planet formation physics. As such, observational programs have already begun using the Hubble Space Telescope (HST, see Madhusudhan \& Seager 2011; Kreidberg et al. 2014; Stevenson et al. 2014; Benneke 2015; Morley et al. 2017; MacDonald \& Madhusudhan 2019) and high-resolution spectroscopy from the ground (Brogi et al. 2013; Birkby et al. 2017) to study the chemical properties of hot Jupiters. Furthermore, interferometric studies of directly imaged planets have begun inferring $\mathrm{C} / \mathrm{O}$ for planets orbiting further from their host star (see e.g. Gravity Collaboration 2020). These characterisation studies have long relied on detecting the most abundant carbon and oxygen carrying molecules (ex. $\mathrm{H}_{2} \mathrm{O}, \mathrm{CO}_{2}, \mathrm{CO}, \mathrm{CH}_{4}$ ) to determine $\mathrm{C} / \mathrm{O}$. Observational programs targeting carbon- and oxygen-bearing species consist mainly in the near-infrared (NIR) and are complicated by the presence of clouds (as discussed in Benneke 2015; Sing et al. 2015, among others). Additionally, overlapping spectral features of $\mathrm{CO}$ and $\mathrm{CO}_{2}$ along with low spectral resolution (Madhusudhan et al. 2011) make determining C/O difficult.

Meanwhile, no observational programs have strongly detected nitrogen-bearing species to determine N/O (but see MacDonald \& Madhusudhan 2017, for a weak detection of $\mathrm{NH}_{3}$ and $\mathrm{HCN}$ ). Given that nitrogen is among the most abundant molecules heavier than helium, its characterisation should help to understand the details of planet formation in the same way that carbon and oxygen have. Unfortunately, its primary molecular species $-\mathrm{N}_{2}-$ is effectively invisible ${ }^{1}$. Neither of the next two most abundant $\mathrm{N}$-bearing species $-\mathrm{NH}_{3}$ and $\mathrm{HCN}-$ are favoured for hot Jupiter atmospheres with solar $\mathrm{C} / \mathrm{O}$. The presence of $\mathrm{NH}_{3}$ is only expected (in equilibrium chemistry calculations) for low $(<500 \mathrm{~K})$ temperatures, while $\mathrm{HCN}$ is favoured only for $\mathrm{C} / \mathrm{O} \geq 1$. In disequilibrium models these molecules can become more abundant due to quenching and photodissociation (Moses et al. 2011), however they never become the primary carrier in hot Jupiter atmospheres.

\footnotetext{
1 It lacks a dipole moment and strong quadrupole moment observable in visible or IR.
} 
In this study we posit that less abundant, metal oxides could be used to infer the bulk chemical properties, especially the nitrogen abundance, of hot Jupiter atmospheres. In particular, we focus on titanium oxide ( $\mathrm{TiO}$ ) which has strong spectral features in the optical, between 0.4 and 1 microns. This fact has made it a popular target for chemical characterisation studies in M- and L-dwarf stars (see e.g. Veyette et al. 2016). Furthermore, it is known that the abundance of titanium-bearing species is strongly linked to the bulk metallicity of M-dwarf stars (Dhital et al. 2012; Kesseli et al. 2019), and given the link between bulk metallicity and C/O (Brewer \& Fischer 2016) it should be no surprise that a connection between $\mathrm{TiO}$ and bulk chemical properties can be made.

The production and destruction of $\mathrm{TiO}$ is discussed in Sect. 2 and relies on the reaction of elemental titanium with generally less abundant nitrogen- and oxygen-bearing molecules. We handle its chemistry using the chemical kinetic code VULCAN along with the chemical network for thelatter two elements, which includes the production of TiO. We expect that their abundance, and the subsequent abundance of $\mathrm{TiO}$ depend sensitively on $\mathrm{C} / \mathrm{H}, \mathrm{O} / \mathrm{H}$, and $\mathrm{N} / \mathrm{H}$, and hence the detection of $\mathrm{TiO}$ can be connected through chemical modelling to the bulk chemical properties of these abundant elemental species in hot Jupiter atmospheres. However, we find that changes in N/H do not translate into changes in $\mathrm{TiO}$ abundance and hence detections of $\mathrm{TiO}$ cannot be directly connected to nitrogen abundances; see Appendix A.

We refer to N/O throughout Sects. 2 and 3 of this work as it relates to our prediction that only the relative abundances of those elements are relevant for the chemistry of TiO. Because it turns out that between nitrogen and oxygen, only oxygen is relevant for the $\mathrm{TiO}$ abundance we switch to reporting only $\mathrm{O} / \mathrm{H}$ (relative to solar) in Sects. 4 and 5 as the latter is more comparable to what can be inferred observationally at this time.

We find that, with the right metric, we can distinguish between different bulk chemical models in the transmission spectra of hot Jupiters. We apply our model to exoplanets with observed transmission spectra covering the necessary wavelength range to deduce whether their inferred $\mathrm{C} / \mathrm{O}$ and $\mathrm{O} / \mathrm{H}$ are consistent with chemical modelling. When not included in the observational studies, we assume a solar value for $\mathrm{C} / \mathrm{O}$ and $\mathrm{O} / \mathrm{H}$. We outline our methods in Sects. 2 and 3, report our results in Sects. 4 and 5, discuss our results in Sect. 6, and conclude in Sect. 7.

\section{Method: atmosphere chemical model}

To model the chemistry occurring in hot Jupiter atmospheres we use the open source chemical kinetic code VULCAN (Tsai et al. 2017). It solves a set of continuity equations for different molecular species, taking into account the production rate, loss rate, and transport flux of each species. The set of equations that VULCAN solves is (Tsai et al. 2017):

$\frac{\partial n_{i}}{\partial t}=\mathcal{P}_{i}-\mathcal{L}_{i}-\frac{\partial \phi_{i}}{\partial z}$

where $n_{i}$ is the number density of the $i$ th species, and $\mathcal{P}_{i}$ and $\mathcal{L}_{i}$ are the reactions that produce and destroy the $i$ th species. The transport term $\phi_{i}$ includes both eddy and molecular diffusion, with the former taking the following form:

$\phi_{i, \text { eddy }}=-K_{z z} n_{\text {total }} \frac{\partial X_{i}}{\partial z}$, where $K_{z z}$ parameterises the strength of mixing in the vertical (z) direction and varies with height. Here, $X_{i}$ is the abundance of the $i$ th species relative to the total number of particles which is denoted by $n_{\text {total }}$. The functional form of the molecular diffusion is taken from Banks \& Kockarts (1973).

The production and destruction rates $\left(k_{i}\right)$ for the $i$ th species depend on the local temperature of the gas, and follow the typical modified Arrhenius equation, with the form:

$k_{i}=A B^{T} \exp (-C / T)$

where the parameters $A, B$, and $C$ are determined from laboratory experiments, computed numerically, or are estimated. The particular chemical network that we used can be found

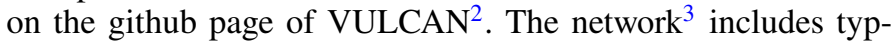
ical high-temperature gas chemistry of carbon-, oxygen-, and nitrogen-bearing molecules, the oxidising reactions of vanadium and titanium (discussed more below), and photodissociation reactions of $\mathrm{H}_{2}, \mathrm{H}_{2} \mathrm{O}, \mathrm{CO}, \mathrm{CO}_{2}, \mathrm{CH}_{3}, \mathrm{CH}_{4}, \mathrm{C}_{2} \mathrm{H}_{2}, \mathrm{C}_{2} \mathrm{H}_{4}, \mathrm{~N}_{2}$, $\mathrm{NH}_{3}$, and $\mathrm{HCN}$.

\subsection{Elemental abundances}

Our chemical model includes the elements: H, He, C, O, N, V, and $\mathrm{Ti}$, and in general we assume solar abundances for each element (relative to $\mathrm{H}$ ). In our search through parameter space, we keep N/H constant while varying the carbon and oxygen abundances to achieve our necessary $\mathrm{C} / \mathrm{O}$ and N/O. As such, our method is sensitive to variations in $\mathrm{C} / \mathrm{O}$ and $\mathrm{O} / \mathrm{H}$. We tested variations in $\mathrm{N} / \mathrm{H}$ in Appendix $\mathrm{A}$ and found no change in the abundance of $\mathrm{TiO}$, discussed below.

The solar abundance of the aforementioned elements are: $\mathrm{He} / \mathrm{H}=0.097, \mathrm{C} / \mathrm{H}=2.78 \times 10^{-4}, \mathrm{O} / \mathrm{H}=4.90 \times 10^{-4}, \mathrm{~N} / \mathrm{H}=$ $8.19 \times 10^{-5}$ (all from Asplund et al. 2009), $\mathrm{V} / \mathrm{H}=9.76 \times 10^{-9}$ and $\mathrm{Ti} / \mathrm{H}=8.43 \times 10^{-8}$ (from Lodders et al. 2009). These elemental abundances result in $\mathrm{C} / \mathrm{O}=0.54$ and $\mathrm{N} / \mathrm{O}=0.17$, the solar values, and are used as a baseline for our chemical models. We sample $\mathrm{C} / \mathrm{O} \in[0.08,1.0]$ and $\mathrm{N} / \mathrm{O} \in[0.01,2]$ for testing the response of $\mathrm{TiO}$ production as a function of bulk chemical composition. We show our sampling of parameter space in Fig. 1.

Our choice of range in $\mathrm{C} / \mathrm{O}$ was selected to mimic the range of $\mathrm{C} / \mathrm{O}$ that is attained from formation models of hot Jupiters. (Cridland et al. 2019b,a). In those models, C/O $>1$ is very difficult to obtain because the gas, ice, and refractory material in protoplanetary discs tend to be more oxygen rich than carbon rich (Mordasini et al. 2014; Cridland et al. 2019b). Furthermore, one might expect that "carbon-rich" planets (with $\mathrm{C} / \mathrm{O}>1$ ) should not have strong spectral features from TiO, as its production should be drastically reduced due to the fact that most of the oxygen in these types of atmospheres should be in $\mathrm{CO}$.

In the aforementioned hot Jupiter formation models, there are minimal (less than $1 \%$ ) differences in the $\mathrm{C} / \mathrm{H}$ and $\mathrm{N} / \mathrm{H}$ of the population of synthetic planets. $\mathrm{O} / \mathrm{H}$ varies by an order of magnitude, which results in N/O variations between 0.01 and 0.1 . We extended the range of N/O for our current experiment to account for variations in $\mathrm{O} / \mathrm{H}$ and $\mathrm{N} / \mathrm{H}$ that might occur between different stars (i.e. in Brewer \& Fischer 2016) ${ }^{4}$. To fill parameter space we allow $\mathrm{C} / \mathrm{H}$ to vary more than was seen in the formation models to

\footnotetext{
2 https://github.com/exoclime/VULCAN

3 TiVNCHO_photo_network_v1025

4 In that work $\mathrm{O} / \mathrm{H}$ varied between 0.6 and $2 \times$ solar however did not include measurements of $\mathrm{N} / \mathrm{H}$.
} 


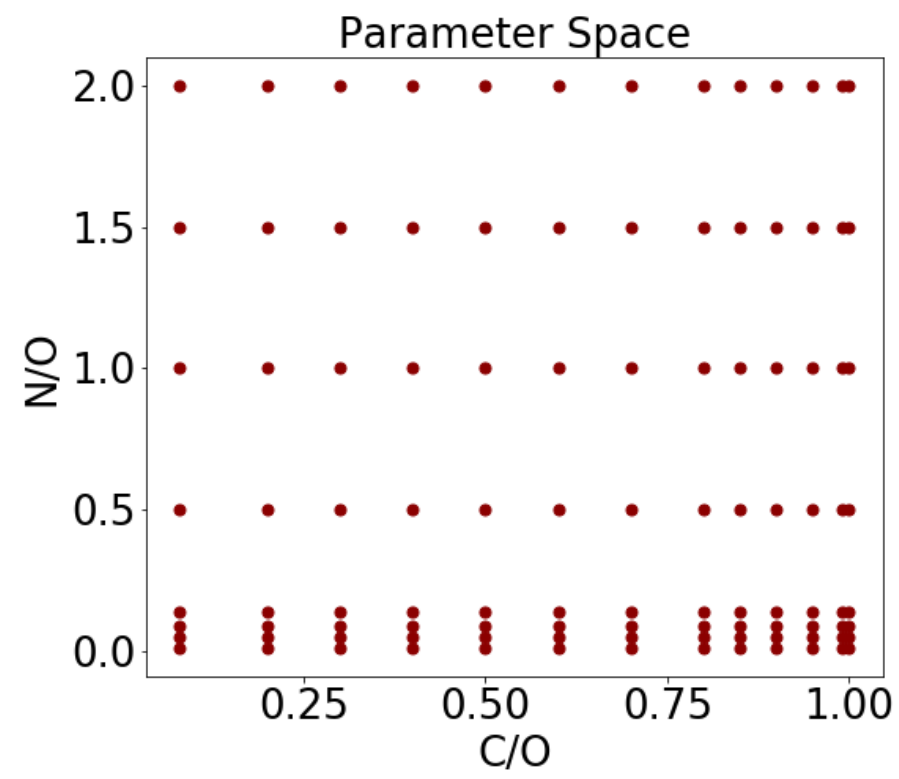

Fig. 1. Parameter space for our chemical modelling simulation. $\mathrm{C} / \mathrm{O}$ and N/O ratios span an order of magnitude across values that are attainable from formation models (e.g. Cridland et al. 2019a).

Table 1. Relevant stellar and planetary properties.

\begin{tabular}{ccc}
\hline \hline & WASP-19b & HD 209458b \\
\hline $\log (g)$ & 3.16 & 2.97 \\
$T_{\text {eq }}(\mathrm{K})$ & 2077 & 2500 \\
$R_{\text {planet }}\left(R_{\text {Jup }}\right)$ & 1.395 & 1.38 \\
$M_{\text {planet }}\left(M_{\text {Jup }}\right)$ & 1.114 & 0.69 \\
Host Type & G8V & G0V \\
$R_{\text {star }}\left(R_{\odot}\right)$ & 1.004 & 1.203 \\
$M_{\text {star }}\left(M_{\odot}\right)$ & 0.904 & 1.148 \\
$L_{\text {star }}\left(L_{\odot}\right)$ & 0.71 & 1.61 \\
\hline
\end{tabular}

similarly account for variations in $\mathrm{C} / \mathrm{H}$ between different stellar systems 5 .

\subsection{Pressure-temperature structure}

We use pre-computed one-dimensional pressure-temperature (PT) curves to model the physical conditions of the atmosphere. These curves were computed using the self-consistent atmospheric petitCODE (Mollière et al. 2015, 2017). In this work we include two models, the first uses the planet properties (mass, radius, surface gravity) of the hot Jupiter HD 209458b, but artificially changes the orbital radius of the planet such that its equilibrium temperature $\left(T_{\text {eq }}\right)$ is $2500 \mathrm{~K}$. This shift in orbital radius changes it classification to an "ultra"-hot Jupiter. We also model the atmospheric structure of the ultra-hot Jupiter WASP$19 \mathrm{~b}$ (with equilibrium temperature of $2077 \mathrm{~K}$ ). We use these ultra-hot Jupiter models to guarantee that $\mathrm{TiO}$ will not condense into clouds, because our chemical kinetic model does not include cloud formation and heavier elements like titanium should prefer to condense in lower temperature atmospheres. We outline the relevant stellar and planetary properties in Table 1.

5 Brewer \& Fischer (2016) find $\mathrm{C} / \mathrm{H}$ variations between 0.3 and $2 \times$ solar.

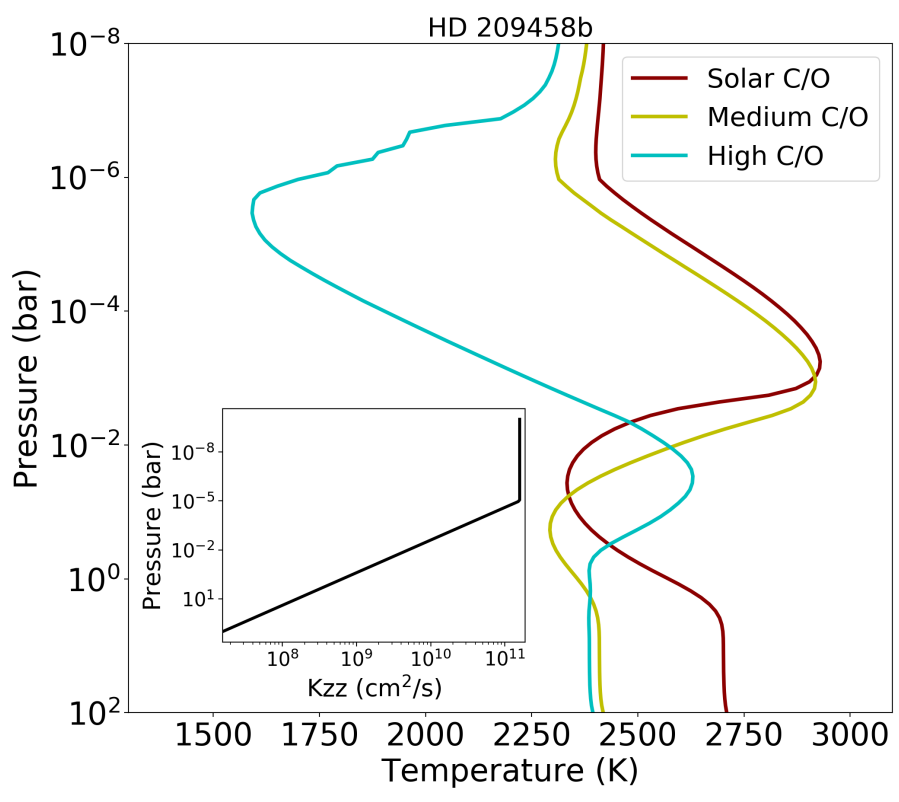

Fig. 2. Pressure-temperature atmospheric profile for the ultra-hot model of HD 209458b. Here we show three models: Solar C/O $(=0.54)$, Medium C/O $(=0.8)$, and High $\mathrm{C} / \mathrm{O}(=1.0)$. As discussed in Mollière et al. (2015), thermal inversion occurs in both temperature profiles, even in the high $\mathrm{C} / \mathrm{O}$ case where $\mathrm{TiO}$ is underabundant. In the inset we show the $K z z$ model used in VULCAN, computed with petitCODE for HD 209458b.

The chemistry used in producing our PT curves is assumed to be in thermochemical equilibrium, meaning that they are generated by minimising the Gibbs free energy of the system. This method is very fast, which allows its inclusion into a self-consistent code like petitCODE. However, this method differs from the method that we use to predict TiO abundances (described above); we use the chemical kinetic solver VULCAN to calculate a kinetic steady state for the chemical system. We acknowledge the possible discrepancy between the chemical structure that is used in deriving the PT curves and the molecular abundances resulting from the chemical kinetic calculation. The base version of VULCAN (ignoring vertical mixing and photodissociation) matches results from the thermochemical equilibrium code TEA (Blecic et al. 2016; Tsai et al. 2017), but including non-equilibrium effects like vertical mixing and photodissociation should bring the chemical system to a steady state that differs from thermochemical equilibrium. We note that our method focuses on data from transmission spectra which are typically less sensitive to the temperature structure than emission spectra.

We compute different PT curves for three different C/O: Solar (=0.54), Medium (=0.8) and High $(=1.0)$. Different $\mathrm{C} / \mathrm{O}$ results in variation in the $\mathrm{H}_{2} \mathrm{O}$ abundance, which can greatly impact the thermal structure in a planetary atmosphere (Mollière et al. 2015). There is a sharp transition in the $\mathrm{H}_{2} \mathrm{O}$ abundance for $\mathrm{C} / \mathrm{O}$ approaching one - hence atmospheres with $\mathrm{C} / \mathrm{O} \lesssim 0.7$ should have similar PT curves (Mollière et al. 2015; Gandhi \& Madhusudhan 2019).

In Fig. 2, we show the PT curves for the ultra-hot version of HD 209458b for the three values of C/O. We note that the PT curves for the Solar and Medium C/O are very similar, which justifies our use of the same PT curve over a wide range of $\mathrm{C} / \mathrm{O}$ when $\mathrm{C} / \mathrm{O}<0.8$. At high $\mathrm{C} / \mathrm{O}$ the abundance of $\mathrm{H}_{2} \mathrm{O}$ has dropped significantly, and the overall temperature of 
the atmosphere is reduced ${ }^{6}$. In the inset of Fig. 2, we show the parameterisation of the vertical mixing, $K_{z z}$. Often this parameter is kept constant throughout the atmosphere, however we choose to use the pressure-dependent $K_{z z}$ computed by petitCODE. As discussed in the Appendix of Mollière et al. (2017), $K_{z z}(\mathrm{P})$ is computed through a combination of mixing length theory (e.g. Kippenhahn \& Weigert 1990) and fitted results from threedimensional circulation models (i.e. $K_{z z} \propto \mathrm{P}^{-1 / 2}$, see Parmentier et al. 2013).

\subsection{TiO production}

Production reactions for $\mathrm{TiO}$ have recently been added to the VULCAN chemical network. These reactions are largely based on lab experimental data where the oxidation of elemental titanium in the gas phase was studied (see Ritter \& Weisshaar 1989, 1990). In their work, Ritter \& Weisshaar (1989) found that $\mathrm{TiO}$ was easily formed by the oxidation of Ti by $\mathrm{NO}, \mathrm{O}_{2}$, and $\mathrm{N}_{2} \mathrm{O}$; however, $\mathrm{TiO}$ was not the end point of their oxidation experiment. They assumed that further oxidation of $\mathrm{TiO}$ takes place producing $\mathrm{TiO}_{2}$. On top of the $\mathrm{TiO}$ reactions in VULCAN, we add two further oxidation reactions to produce $\mathrm{TiO}_{2}$ based on the kinetic data generated by Ritter \& Weisshaar (1989). The full set of $\mathrm{TiO}$ reactions are:

$$
\begin{aligned}
& \mathrm{Ti}+\mathrm{NO} \rightleftharpoons \mathrm{TiO}+\mathrm{N} \\
& \mathrm{Ti}+\mathrm{O}_{2} \rightleftharpoons \mathrm{TiO}+\mathrm{O} \\
& \mathrm{Ti}+\mathrm{N}_{2} \mathrm{O} \rightleftharpoons \mathrm{TiO}+\mathrm{N}_{2} \\
& \mathrm{TiO}+\mathrm{NO} \rightleftharpoons \mathrm{TiO}_{2}+\mathrm{N} \\
& \mathrm{TiO}+\mathrm{O}_{2} \rightleftharpoons \mathrm{TiO}_{2}+\mathrm{O} .
\end{aligned}
$$

As mentioned above, the oxidisers responsible for the production of $\mathrm{TiO}$ are generally underabundant in chemical models of hot Jupiter atmospheres. Hence, we expect that the generation and maintenance of $\mathrm{TiO}$ will depend on the bulk chemical composition of the exoplanetary atmospheres.

In Fig. 3, we compare the abundances of the main oxygen carriers with the molecules responsible for the generation of TiO. Here, we see that these oxidisers are at least two orders of magnitude less abundant than the main oxygen carriers. They also vary by between one and two orders of magnitude over all pressures when $\mathrm{C} / \mathrm{O}$ is varied by a factor of four. To reiterate, due to these variations we expect the abundance of TiO to vary as we change $\mathrm{C} / \mathrm{O}$.

\subsection{Typical chemical structure}

Generally speaking, ultra-hot Jupiters orbit at a typical radius where one might expect photodissociation to dominate the chemical abundances in the upper atmosphere, driven by a high flux of ultra-violet (UV) light. We assume a solar-like UV spectrum (based on a PHOENIX model with $T_{\text {eff }}=5700 \mathrm{~K}$ ) to dictate the flux of UV photons at a given wavelength, and used the photodissocation reactions that are included in the updated version of VULCAN. These reactions include the photodissociation of $\mathrm{H}_{2} \mathrm{O}, \mathrm{CO}, \mathrm{CO}_{2}, \mathrm{H}_{2}, \mathrm{CH}_{4}, \mathrm{CH}_{3}, \mathrm{C}_{2} \mathrm{H}_{2}, \mathrm{C}_{2} \mathrm{H}_{4}, \mathrm{~N}_{2}, \mathrm{NH}_{3}$ and $\mathrm{HCN}$.

Because of strong photodissociation, the chemistry structure of the atmosphere can typically be thought of as being made up of three regions: chemical equilibrium, equilibrium-mixing, and radiation-dominated. The first region occurs low in the

\footnotetext{
6 We note that petitCODE does not include the opacity sources from atomic metal species (e.g. Fe and Ti, Lothringer et al. 2020) which could act as important absorbers and heating sources in the upper atmosphere of ultra hot Jupiters.
}
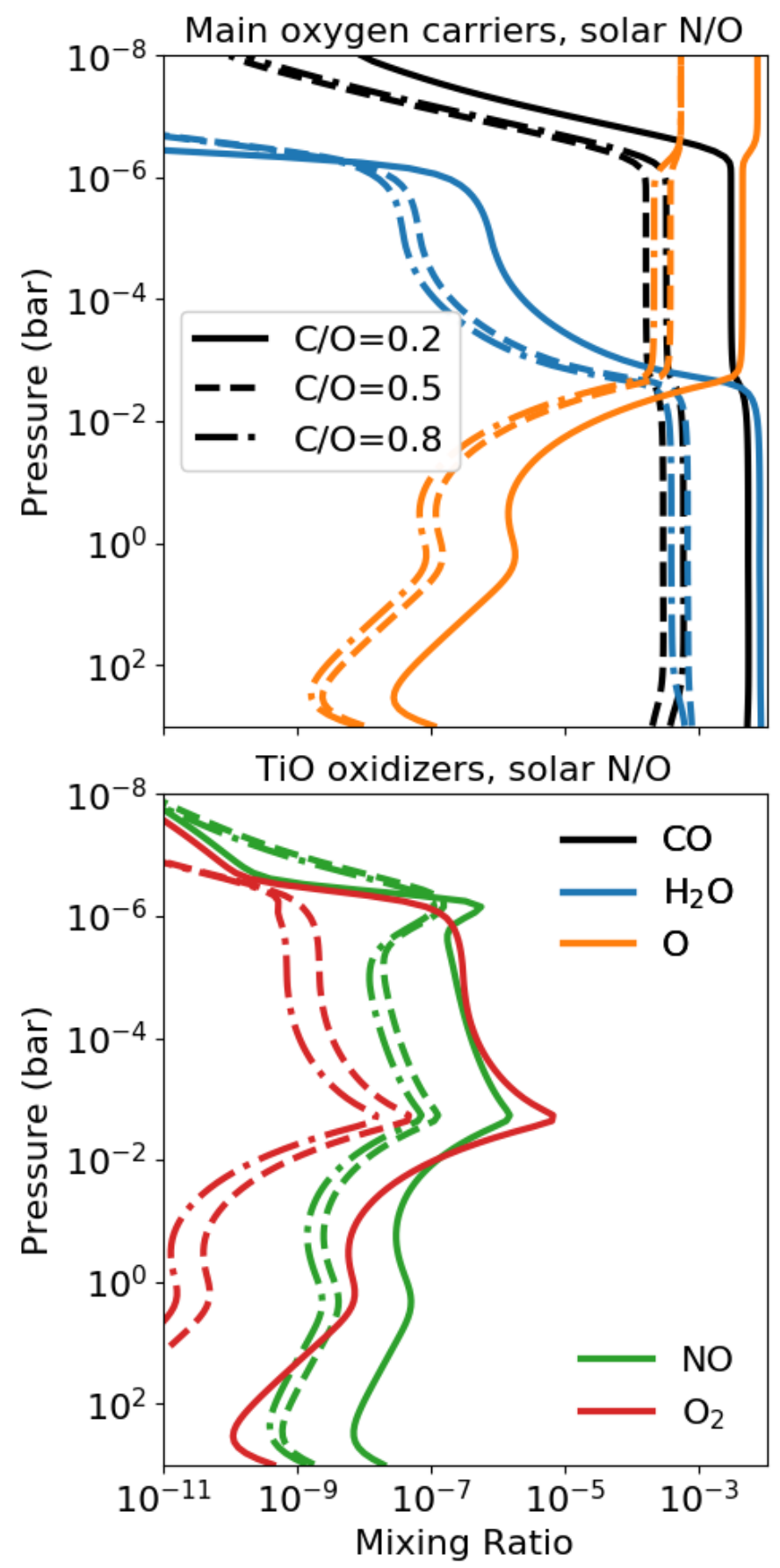

Fig. 3. Example of the chemical distribution of the main oxygen carriers in our chemical models. These species are at least two orders of magnitude more abundant than the oxidisers responsible for the production of $\mathrm{TiO}$. The third $\mathrm{TiO}$ oxidiser, $\mathrm{N}_{2} \mathrm{O}$, is not sufficiently abundant to appear on this figure.

atmosphere $(P>1$ bar), where the dissociating photons cannot reach the gas and the solution to the chemical model is similar to the thermochemical solution. The second region occurs between $10^{-3}$ and 1 bar, and represents a combination of chemical equilibrium and vertical mixing dictating the abundance of molecular species. In this region we see a fast reduction in $\mathrm{H}_{2} \mathrm{O}$ as it is thermally dissociated into atomic $\mathrm{O}$ and $\mathrm{H}$ (see Fig. 3), but more stable molecules like $\mathrm{CO}$ tend to remain abundant. Very high up in the atmosphere $\left(P<10^{-3}\right)$, photodissociation dominates the chemical evolution, and the densities are sufficiently 

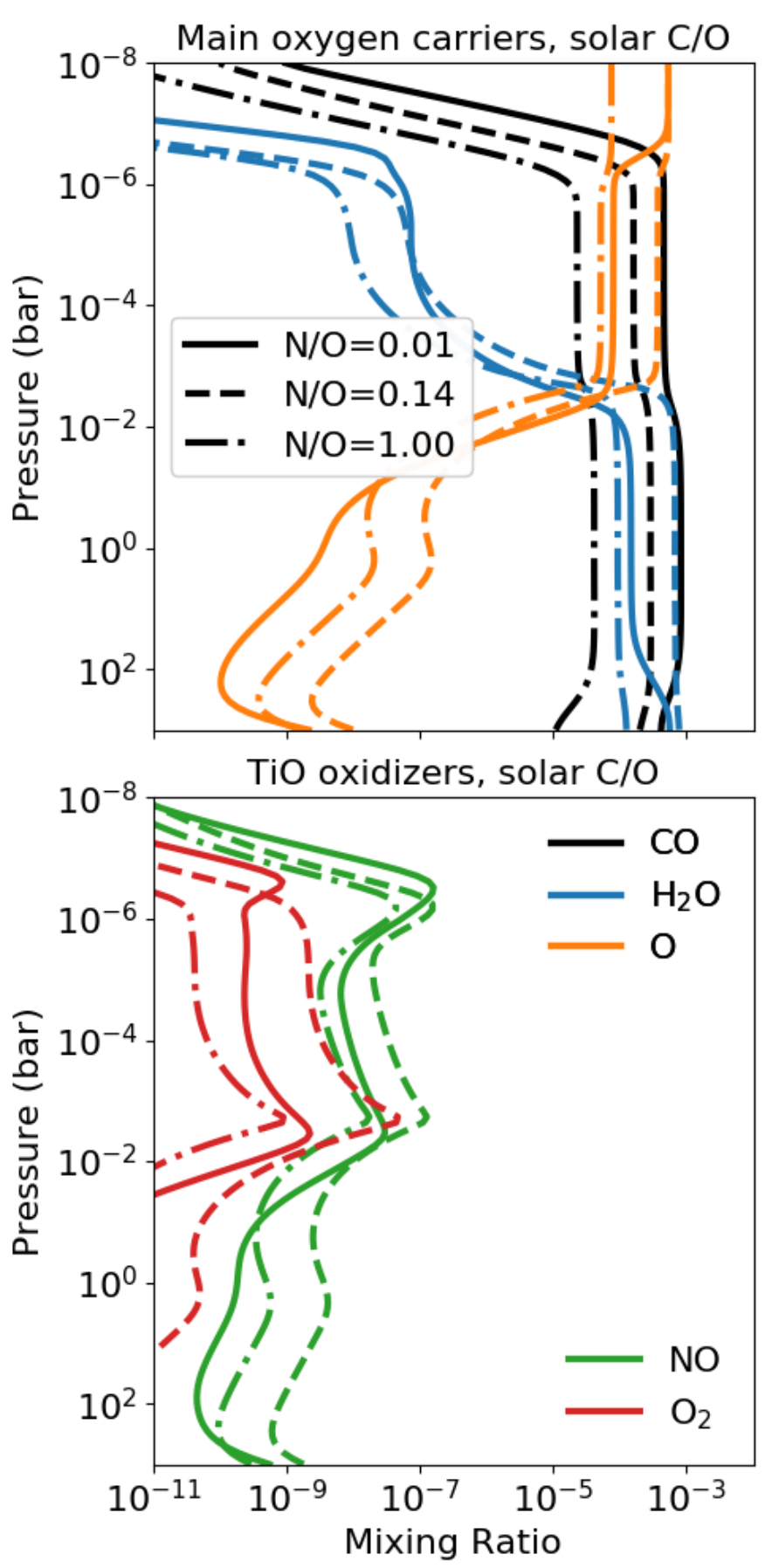

Fig. 4. Example of the chemical distribution of the main oxygen carriers in our chemical models as a function of N/O. Similar to Fig. 3, increasing N/O reduces the oxygen abundance available for the production of oxygen-carrying species.

low that recombination is inefficient. In this third region, even the most stable molecules are dissociated, leaving only atomic species in the upper atmosphere.

In Fig. 4 we show a similar comparison to Fig. 3, except for variations in $\mathrm{N} / \mathrm{O}$ with a constant solar $\mathrm{C} / \mathrm{O}$. Unlike in Fig. 3, where increases in $\mathrm{C} / \mathrm{O}$ result in monotonic decreases in $\mathrm{TiO}$ oxidising species, there appears to be an optimal ratio of nitrogen to oxygen $(=0.14)$ that results in the highest $\mathrm{TiO}$ oxidiser abundances. In our chemical model, we varied N/O by adjusting $\mathrm{O} / \mathrm{H}$ while keeping $\mathrm{N} / \mathrm{H}$ constant. We checked what effect changing $\mathrm{N} / \mathrm{O}$ through variations in $\mathrm{N} / \mathrm{H}$ had on the resulting
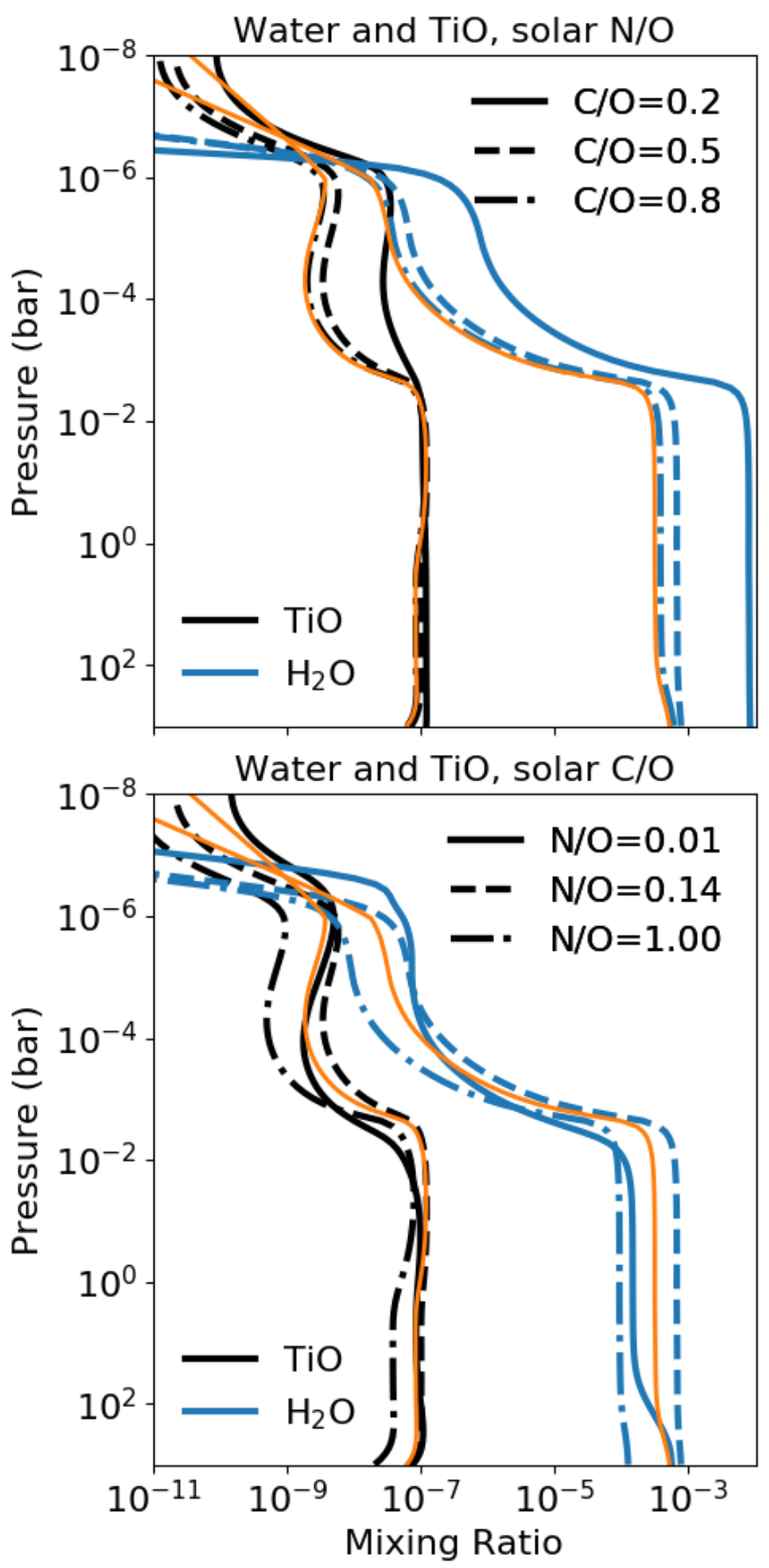

Fig. 5. Variation of $\mathrm{TiO}$ and $\mathrm{H}_{2} \mathrm{O}$ abundance as a function of both the $\mathrm{C} / \mathrm{O}$ (top) and N/O (bottom). The abundance of TiO is most sensitive in the region of the atmosphere which is determined by a combination of vertical mixing and photodissociation. For comparison we show the abundances for solar $\mathrm{C} / \mathrm{O}$ and N/O assuming thermochemical equilibrium with orange lines - these should be compared to the dashed lines in both panels.

abundance of TiO; this test is presented in Appendix A. We find that an increase in $\mathrm{N} / \mathrm{H}$ is not equivalent to a similar decrease in $\mathrm{O} / \mathrm{H}$ and therefore, in what follows, variations in N/O should be interpreted primarily as variations in $\mathrm{O} / \mathrm{H}$.

We show in Fig. 5 how these small changes in the abundance of $\mathrm{TiO}$ oxidisers impact the steady-state abundance of $\mathrm{TiO}$ in the atmospheres of the ultra-hot Jupiters. Generally, the largest changes are found in the region of the atmosphere that 


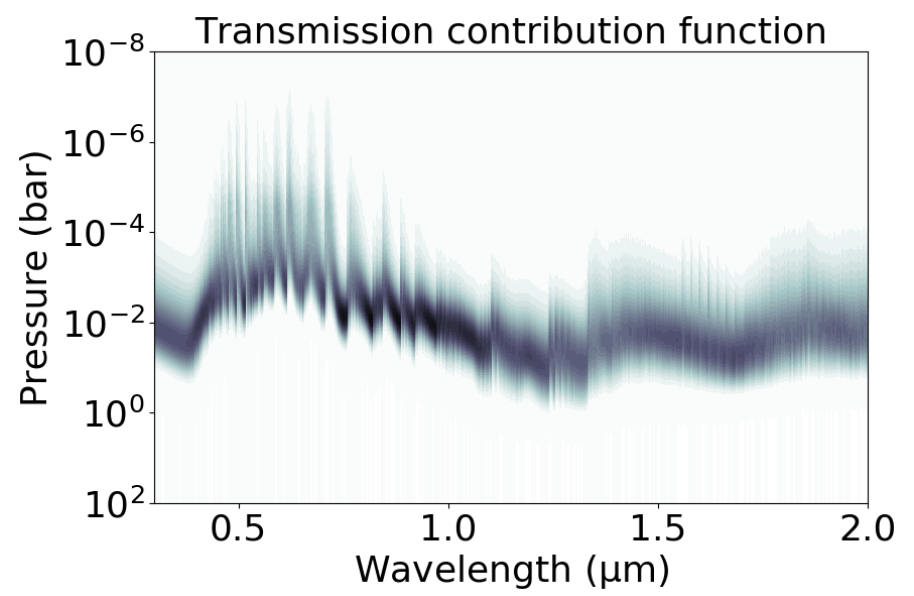

Fig. 6. Contribution function for the synthetic spectra of the ultra hot version of $\mathrm{HD} 209458 \mathrm{~b}$ in the case of a high $\mathrm{C} / \mathrm{O}$ ratio. The $\mathrm{TiO}$ feature primarily comes from the upper levels in the atmosphere, in the region of the atmosphere that depends on both photodissociation and vertical mixing. On the other hand, the $\mathrm{H}_{2} \mathrm{O}$ feature originates from deeper in the atmosphere, overlapping the chemical equilibrium region.

depends on the combination of vertical mixing and chemical equilibrium. We compare our results to the abundances of $\mathrm{TiO}$ and $\mathrm{H}_{2} \mathrm{O}$ derived by thermochemical equilibrium calculations (in orange). We see only small variation between those models and the solar elemental ratio models shown in Fig. 5 (dashed lines). The largest deviations are found in the very upper atmosphere where photodissociation destroys the water that should otherwise be found there. Below, we investigate whether these differences in abundance caused by the bulk chemical properties can be detected by studying the transmission spectra of hot Jupiters.

\section{Method: synthetic spectra}

To compare to observations, we generate synthetic spectra for each of our modelled planets using our chemical models as inputs for petitRADTRANS (Mollière et al. 2019) ${ }^{7}$. This radiative transfer scheme assumes that the 1D profile shown in the last section dictates the chemical distribution over all observable latitudes. The code then draws lines of sight through the atmosphere and computes the wavelength-dependent optical depth along these lines of sight. With these optical depths, the code produces a transmission spectrum which describes the apparent size of the planet as it transits in front of its host star.

As the light ray passes through the planetary atmosphere, it passes through different levels of pressures, densities, and as such abundances. In Fig. 6, we show the contribution function for a typical chemical model of HD 209458b. This function describes from what region in the atmosphere the transmission signal originates. Generally speaking, the $\mathrm{TiO}$ signature (between 0.4 and $0.8 \mu \mathrm{m}$ ) originates from higher in the atmosphere than the $1.4 \mu \mathrm{m} \mathrm{H}_{2} \mathrm{O}$ feature.

The species that we consider to generate the synthetic spectra are: $\mathrm{H}_{2}, \mathrm{H}_{2} \mathrm{O}, \mathrm{OH}, \mathrm{CO}, \mathrm{CO}_{2}, \mathrm{CH}_{4}, \mathrm{C}_{2} \mathrm{H}_{2}, \mathrm{HCN}, \mathrm{NH}_{3}, \mathrm{TiO}$ and VO. We also consider Rayleigh scattering for $\mathrm{H}_{2}$ and $\mathrm{He}$, as well as collision induced absorption for the $\mathrm{H}_{2}-\mathrm{H}_{2}$ and $\mathrm{H}_{2}-\mathrm{He}$ pairs. The molecular abundances computed by VULCAN in one dimension were used as inputs for petitRADTRANS.

https://gitlab.com/mauricemolli/petitRADTRANS

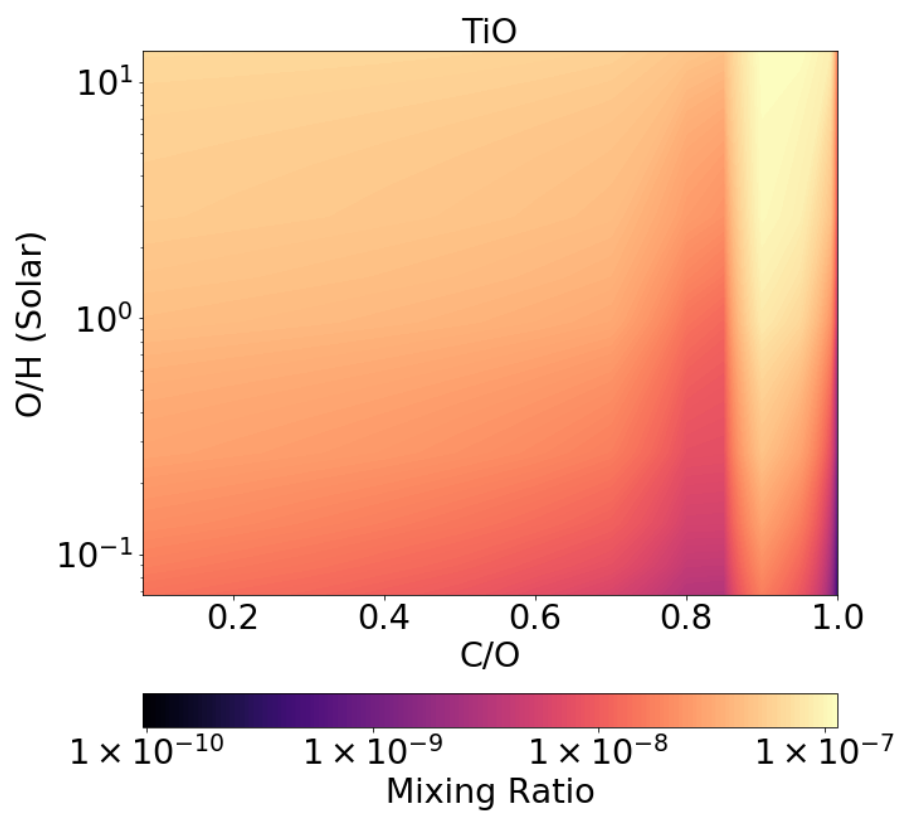

Fig. 7. Abundance of $\mathrm{TiO}$ as a function of $\mathrm{C} / \mathrm{O}$ and $\mathrm{O} / \mathrm{H}$ for the ultra-hot model of HD 209458b, averaged through a pressure range of $10^{-4}$ and $10^{-2}$ bar. There is a smooth decrease in $\mathrm{TiO}$ abundance for increasing $\mathrm{C} / \mathrm{O}$ and decreasing $\mathrm{O} / \mathrm{H}$ until we switch our PT curve to the "High C/O" model.

For calculating synthetic spectra, we need to define the planetary radius, its gravity and pressure (at the defined planetary radius), the PT profile, the mean molecular weight, and the chemical abundance, in units of mass fractions (the former two calculated from VULCAN).

\section{Results: exploring $\mathrm{C} / \mathrm{O}$ and $\mathrm{O} / \mathrm{H}$ parameter space}

\subsection{Dependence of TiO abundance}

Before directly comparing our synthetic spectra to observations, we first explore the impact of the bulk chemical composition on the abundance of TiO. In Fig. 7, we show a colour map for the abundance of $\mathrm{TiO}$ varying both $\mathrm{C} / \mathrm{O}$ and $\mathrm{O} / \mathrm{H}$ ratios. The abundance that we show is an average across the pressure range of $10^{-4}-10^{-2}$ bar, which we assume describes the abundance that will most impact the observed $\mathrm{TiO}$ features from transmission spectra (recall Fig. 6).

We find a smooth transition in the abundance of $\mathrm{TiO}$ for $\mathrm{C} / \mathrm{O}=0.8$, with the abundance reducing for increasing $\mathrm{C} / \mathrm{O}$ and decreasing $\mathrm{O} / \mathrm{H}$. Above $\mathrm{C} / \mathrm{O}=0.85$, we change our PT curve to the "High C/O" structure shown in Fig. 2, which results in the discontinuity of $\mathrm{TiO}$ abundance that occurs for models with $\mathrm{C} / \mathrm{O}=0.85$. For models with $\mathrm{C} / \mathrm{O}>0.85$, the abundance of $\mathrm{TiO}$ again reduces for increasing $\mathrm{C} / \mathrm{O}$ with the abundance dropping significantly at $\mathrm{C} / \mathrm{O}=1$.

Our result of higher $\mathrm{TiO}$ abundances for $\mathrm{C} / \mathrm{O}>0.85$ was unexpected, as equilibrium chemical calculations with high $\mathrm{C} / \mathrm{O}$ generally result in low $\mathrm{TiO}$ abundance. This discrepancy can be understood in context of the chemical models of Lothringer et al. (2018), who compute $\mathrm{TiO}$ abundances over a wide range of pressures and temperatures. For pressures between $10^{-4}$ and $10^{-2}$ bar, our Solar- and Medium-C/O atmospheric models have gas temperatures between 2500 and $2800 \mathrm{~K}$. At these temperatures, Lothringer et al. (2018) found that equilibrium abundances of $\mathrm{TiO}$ were depleted by an order of magnitude because of 


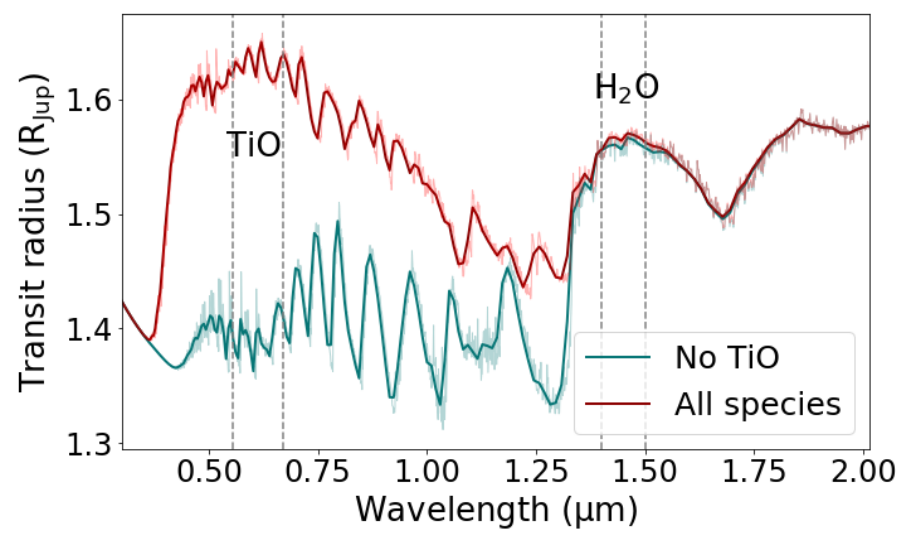

Fig. 8. Transmission spectrum for the ultra-hot model of HD 209458b. We consider all the species in our chemical model (red line) and compare to a sample spectrum derived for this model but with $\mathrm{TiO}$ removed (blue line). The vertical dashed lines denote particular wavelength ranges that we use in our analysis to compare to observations.

thermal dissociation. In our High-C/O model, the temperatures in the mentioned pressure range are between 1800 and $2400 \mathrm{~K}$ because there is no high thermal inversion in that model. As a result, the chemical system is sufficiently warm that $\mathrm{TiO}$ does not condense into clouds, but cold enough that $\mathrm{TiO}$ is not thermally dissociated. This stability against thermal dissociation in the higher atmosphere preserves $\mathrm{TiO}$ even in chemical models with $\mathrm{C} / \mathrm{O}=1$, where equilibrium models would otherwise predict significantly lower abundances.

\subsection{Implication for transmission spectra}

$\mathrm{TiO}$ has a large number of electronic transitions in the optical $(0.4-0.8 \mu \mathrm{m})$ that can easily be detected in exoplanetary atmospheres. In Fig. 8, we compare a transmission spectrum for our model HD 209458b atmosphere with and without TiO. There is a very clear difference between these spectra when we remove TiO. Additionally, Fig. 8 shows the wavelength range that we sample to produce the average transmission signal strength used in the following analysis (vertical dashed lines).

To compare our chemical models to observations, we average the signal between the vertical dashed lines shown in Fig. 8. We then compare the difference in the signal strength due to $\mathrm{TiO}\left(Z_{\mathrm{TiO}}\right)$ with the signal strength caused by $\mathrm{H}_{2} \mathrm{O}\left(Z_{\mathrm{H}_{2} \mathrm{O}}\right)$. This difference is normalised by the equilibrium scale height of the atmosphere $\left(H_{\mathrm{eq}}=k T_{\mathrm{eq}} / \mu \mathrm{g}\right.$, where $\mu$ is the mean molecular weight of atmospheric particles, assumed to be 2.3 times the mass of the proton, and $g$ the gravity) to act as a metric for molecular abundances in the atmosphere (see e.g. Sing et al. 2015).

The reason for deriving the metric in this way is as follows: the effective altitude of the atmosphere that is probed by a wavelength $\lambda$ in transmission is estimated by (Lecavelier Des Etangs et al. 2008):

$Z(\lambda)=H_{\mathrm{eq}} \ln \left(\xi_{\mathrm{abs}} P_{z=0} \sigma_{\mathrm{abs}}(\lambda) / \tau_{\mathrm{eq}} \times \sqrt{2 \pi R_{\mathrm{p}} / k T \mu g}\right)$,

where $\sigma_{\mathrm{abs}}$ and $\xi_{\text {abs }}$ is the cross section and abundance of the dominant absorbing species, $P_{z=0}$ is the pressure at the reference altitude, $\tau_{\text {eq }}$ is the optical thickness at the effective radius, and $R_{\mathrm{p}}$ is the planetary radius. The atmospheric scale height is derived for the equilibrium temperature for the planet and the surface gravity. In this way, normalising by $H_{\mathrm{eq}}$ removes from the metric a dependence on the mass and radius of the planet, allowing for a wide range of planets to compare. For two different species (i.e. $\mathrm{TiO}$ and $\mathrm{H}_{2} \mathrm{O}$ ) we then have:

$\frac{\Delta Z_{\mathrm{TiO}-\mathrm{H}_{2} \mathrm{O}}}{H_{\mathrm{eq}}} \equiv \frac{Z_{\mathrm{TiO}}-Z_{\mathrm{H}_{2} \mathrm{O}}}{H_{\mathrm{eq}}}=\ln \left[\frac{\xi_{\mathrm{abs}, \mathrm{TiO}} \sigma_{\mathrm{abs}, \mathrm{TiO}}}{\xi_{\mathrm{abs}, \mathrm{H}_{2} \mathrm{O}} \sigma_{\mathrm{abs}, \mathrm{H}_{2} \mathrm{O}}}\right]$.

We note that this metric depends on both the relative abundance of molecules and their cross section at the selected wavelengths. The latter only weakly changes between models since different lines of sight probe different pressure and temperature ranges resulting in slight changes to cross sections. Comparing this metric between models and observations is most strongly dependent on the relative abundances along a line of sight. Such a comparison can be made between different planets and chemical models since the terms related to the physical properties of the planet are removed.

In Fig. 9, we show different synthetic spectra that result from different chemical models with varying $\mathrm{C} / \mathrm{O}$ ratio for a constant solar $\mathrm{O} / \mathrm{H}$. The different $\mathrm{C} / \mathrm{O}$ s are denoted by the changing colour scale, with darker colours representing increasing $\mathrm{C} / \mathrm{O}$. While only small changes in the strength of the $\mathrm{TiO}$ feature occur up to $\mathrm{C} / \mathrm{O}=0.8$, the water features become increasingly weak. However, once the physical structure of the atmosphere is changed, the water feature becomes a stronger transmission feature while the $\mathrm{TiO}$ feature begins to be suppressed. The jump in features is related to the change in the physical structure, since the colder upper atmosphere results in a lower scale height. The lower scale height and temperature produces higher densities higher in the atmosphere, allowing for an apparently larger water transmission signal. For models with high $\mathrm{C} / \mathrm{O}$, the specific structure of the water feature tends to flatten out since the abundance of the molecule is reduced in these models.

Similarly, in Fig. 10 we show the variation in the transmission spectra due to changes in $\mathrm{O} / \mathrm{H}$, for solar $\mathrm{C} / \mathrm{O}$. In this case, the strength of the $\mathrm{TiO}$ feature is nearly independent of $\mathrm{O} / \mathrm{H}$, which comes from the fact that the abundance of $\mathrm{TiO}$ does not vary strongly with $\mathrm{O} / \mathrm{H}$. However, as the $\mathrm{H}_{2} \mathrm{O}$ feature is strongly dependent on $\mathrm{O} / \mathrm{H}$, the difference in the height of the transmission spectral features will still act as a good metric for determining the bulk properties. While we test $\mathrm{O} / \mathrm{H}$ depletion by up to a factor of two (N/O enhancement of a factor of two), it is unclear (both observationally and theoretically) whether these elemental ratios could indeed be this large.

In both figures, we can see that the relative heights of the $\mathrm{TiO}$ and $\mathrm{H}_{2} \mathrm{O}$ features vary as we change the bulk chemical properties of the atmosphere. We choose to quantify our analysis by using the metric mentioned above, as it should encode the necessary information that would be available to low-resolution chemical characterisation studies of transiting hot Jupiters.

\section{Results: comparing models to observations}

In Fig. 11, we plot $\Delta Z_{\mathrm{TiO}-\mathrm{H}_{2} \mathrm{O}} / H_{\text {eq }}$ as a function of $\mathrm{C} / \mathrm{O}$ and $\mathrm{O} / \mathrm{H}$ ratios for our HD $209458 \mathrm{~b}$ model, as well as the set of models computed with the planetary parameters for WASP-19b. The colour of the points denote the models' $\mathrm{O} / \mathrm{H}$, while their position on the $x$-axis denotes their $\mathrm{C} / \mathrm{O}$. We note that in general these points are well separated in the Solar and Medium C/O models, while these points are less separated in the High $\mathrm{C} / \mathrm{O}$ model. Similarly, the results from separate atmospheric models (HD 209458b and WAPS-19b) give nearly the same value for the metric in Eq. (5), confirming that this metric should remain consistent while studying a wide range of planetary atmospheres. 


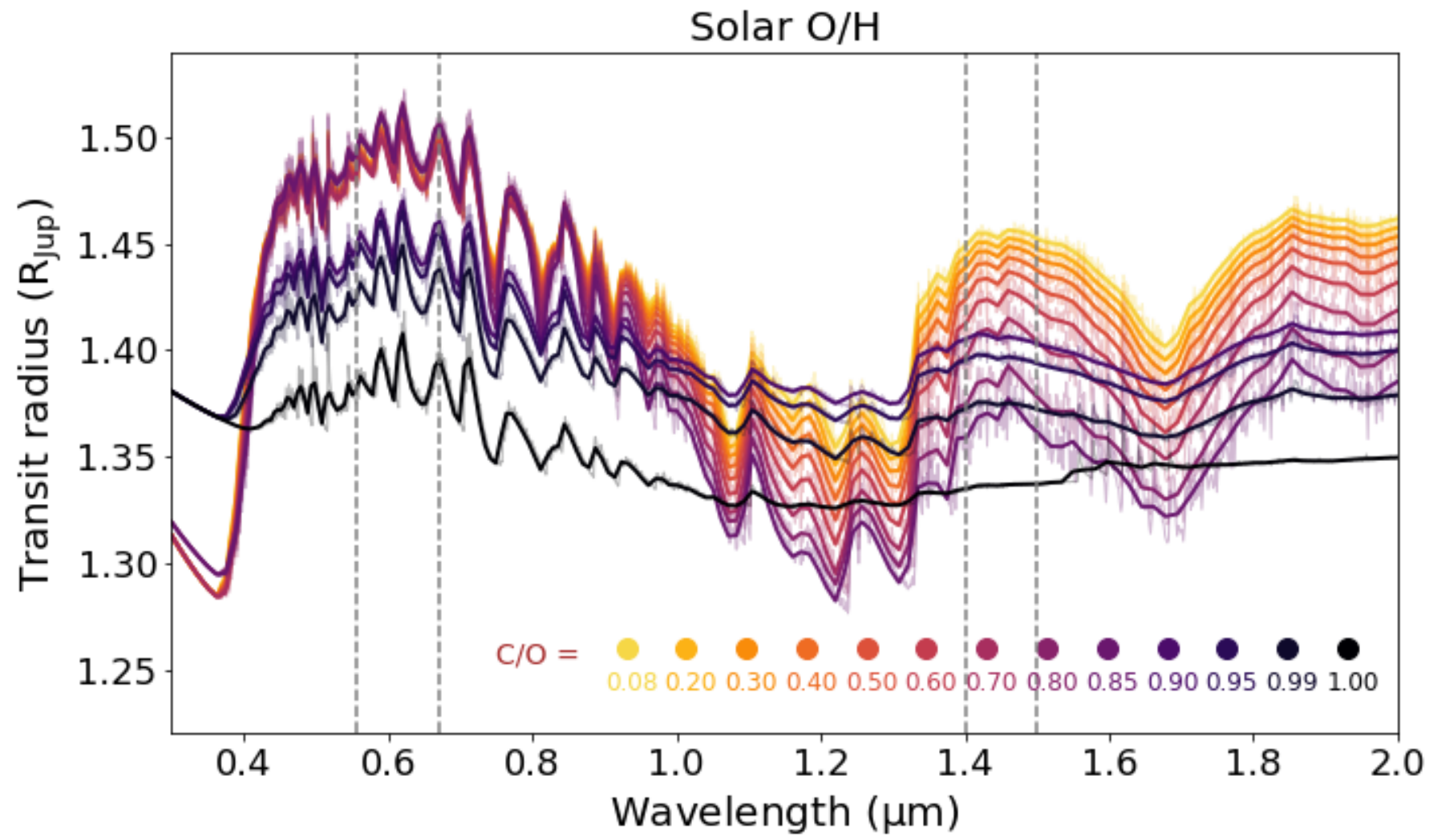

Fig. 9. Transmission spectra for the ultra-hot model of HD $209458 \mathrm{~b}$ as a function of C/O. The spectra are first computed at high resolution and are then convolved to a resolution $\lambda / \Delta \lambda=100$. We leave the high-resolution spectra as faded curves in the background.

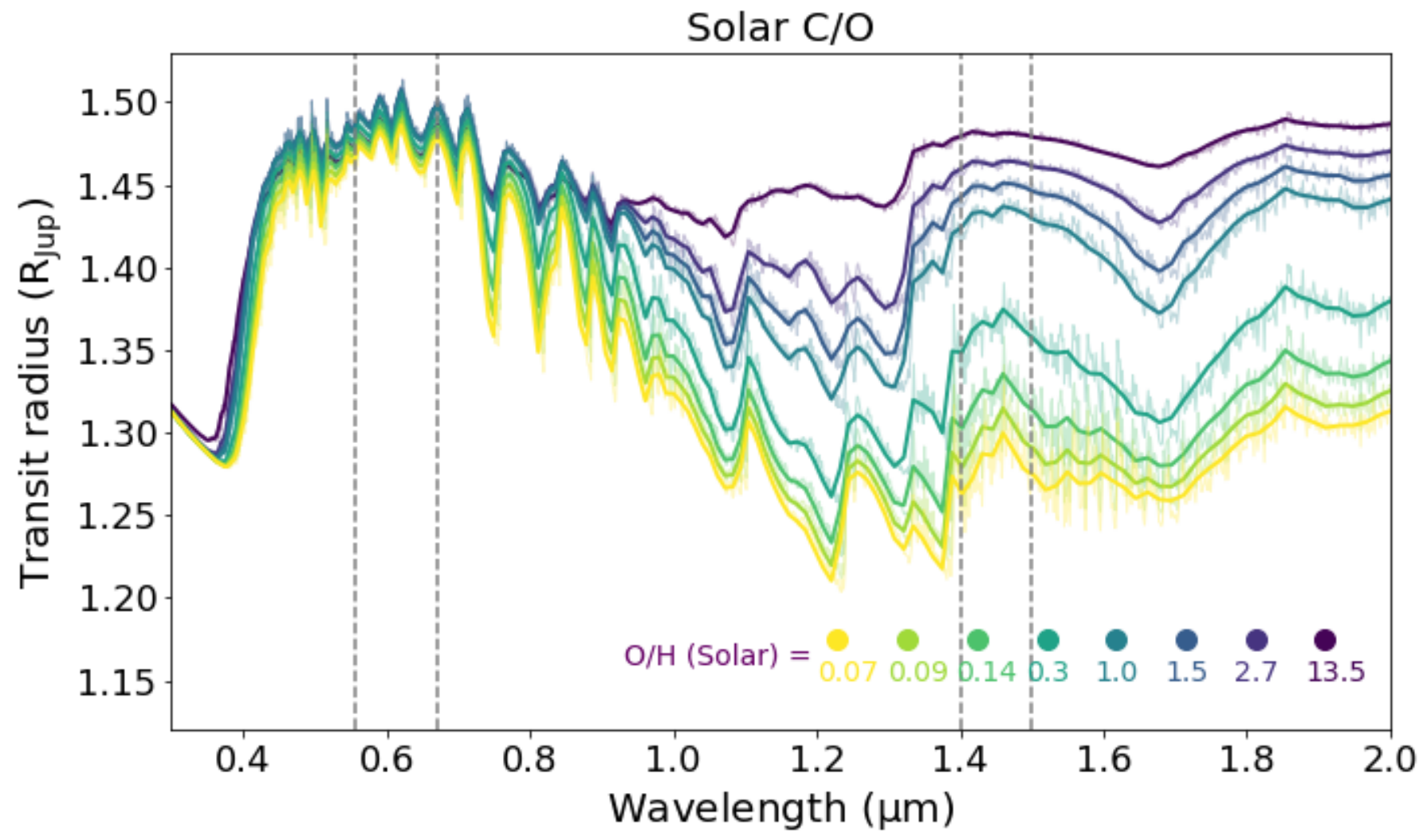

Fig. 10. Same as Fig. 9 but as a function of $\mathrm{O} / \mathrm{H}$. 


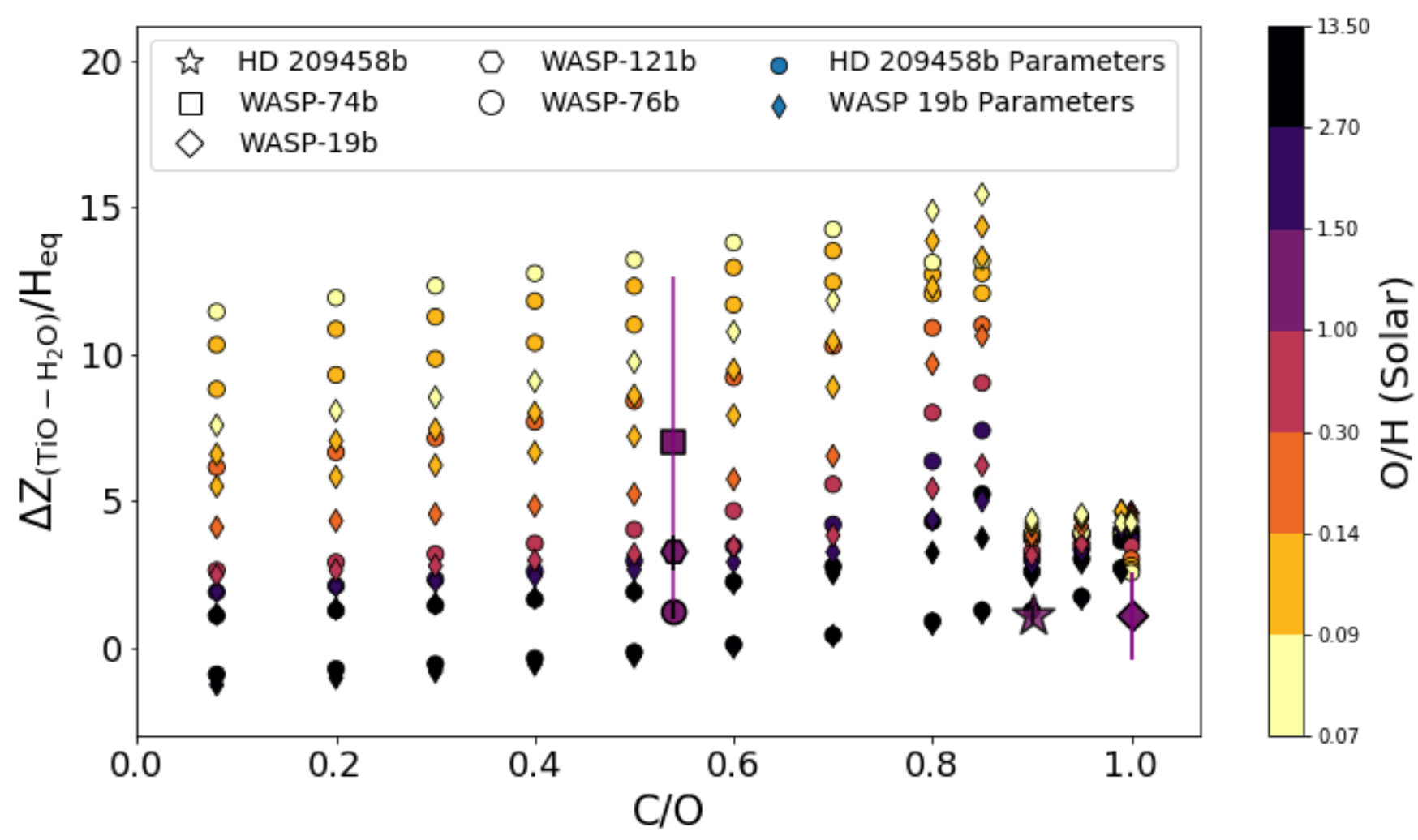

Fig. 11. Abundance metric for $\mathrm{TiO}$ as a function of $\mathrm{C} / \mathrm{O}$ and $\mathrm{O} / \mathrm{H}$ ratios. For $\mathrm{C} / \mathrm{O} \geq 0.9$, the lower temperatures at lower pressures compress the upper atmosphere. This compression, when normalised by $H_{\mathrm{eq}}$ (which is unaffected by $\mathrm{C} / \mathrm{O}$ ) results in the rapid shrink in the metric. The circles and thin diamonds are our physical and chemical models which are compared with observations of hot Jupiters HD 209458b (star; Désert et al. 2008), WASP-74b (square; Mancini et al. 2019), WASP-19b (diamond; Huitson et al. 2013; Sedaghati et al. 2017), WASP-121b (hexagon; Evans et al. 2016), and WASP-76b (octagon; Fu et al. 2020).

As seen with the raw spectra, the difference in the height of the $\mathrm{TiO}$ and $\mathrm{H}_{2} \mathrm{O}$ features increases as $\mathrm{C} / \mathrm{O}$ increases for lower values of $\mathrm{C} / \mathrm{O}$. This trend changes abruptly when we change to the High-C/O model, as the resulting PT curve has lower temperatures at low pressures. This abrupt change is expected from chemical and physical models of exoplanetary atmospheres (as seen in Mollière et al. 2015; Gandhi \& Madhusudhan 2019). With this High $\mathrm{C} / \mathrm{O}$ model, the specific features of both $\mathrm{TiO}$ and $\mathrm{H}_{2} \mathrm{O}$ are suppressed and the difference between their signal strength becomes effectively degenerated with $\mathrm{O} / \mathrm{H}$.

In the Solar and Medium C/O atmospheric models, decreasing $\mathrm{O} / \mathrm{H}$ will increase the difference in strength between the transmission features of $\mathrm{TiO}$ and $\mathrm{H}_{2} \mathrm{O}$. As we saw in Fig. 10, this change in signal comes from a reduction in the strength of the $\mathrm{H}_{2} \mathrm{O}$ signal as $\mathrm{O} / \mathrm{H}$ decreases rather than a change in the $\mathrm{TiO}$ abundance. Regardless, the metric from Eq. (5) is a strong tracer of the $\mathrm{TiO} / \mathrm{H}_{2} \mathrm{O}$ abundance ratio, which will help to constrain the oxygen abundance in exoplanetary atmospheres.

We compare our models with optical/NIR observations of ultra-hot/hot Jupiter atmospheres. For some of these planets, there are direct signals of $\mathrm{TiO}$ which are used to derive the abundance of molecules in their atmospheres, as is the case for WASP-19b (Huitson et al. 2013; Sedaghati et al. 2017), WASP74b (Mancini et al. 2019), and WASP-121b (Evans et al. 2016). While in HD 209458b, Désert et al. (2008) inferred an upper limit for the $\mathrm{TiO}$ abundance after removing the transmission signal coming from strong sodium and potassium lines. Below, we outline comparisons between the inferred chemical properties of these planets and our chemical models. When the oxygen abundance is not presented we assume a solar $\mathrm{O} / \mathrm{H}$.

\subsection{WASP-121b}

Evans et al. (2016) studied the atmosphere of WASP-121b with the Hubble Space Telescope, and directly detected $\mathrm{TiO}$ and $\mathrm{H}_{2} \mathrm{O}$ absorption. These latter authors report an abundance ratio of $\mathrm{TiO} / \mathrm{H}_{2} \mathrm{O}$ of approximately seven times solar relative to results of thermochemical equilibrium calculations, but do not report specific $\mathrm{C} / \mathrm{O}$ and $\mathrm{O} / \mathrm{H}$. If we assume that both $\mathrm{C} / \mathrm{O}$ and $\mathrm{O} / \mathrm{H}$ are solar, then their reported transmission signal is consistent with our synthetic spectra results. This consistency suggests that the non-detection of $\mathrm{TiO}$ (replaced with VO) reported by Evans et al. (2016) can be explained by non-equilibrium processes like vertical mixing or photodissociation - which we include in our chemical model - rather than a variation in the bulk chemical properties away from solar.

We note that the work of Evans et al. (2016) is an excellent observational experiment for our purposes, as it provided a wide wavelength range which included wavelengths relevant for both $\mathrm{TiO}$ and $\mathrm{H}_{2} \mathrm{O}$ absorption. More observations of this kind would greatly improve our comparisons to observations. Interestingly, further observations of this ultra-hot Jupiter have brought into question the existence of $\mathrm{TiO}$ in its atmosphere. Both Evans et al. (2018) and Merritt et al. (2020) report non-detections for $\mathrm{TiO}$ in new HST transmission spectra and high-resolution cross-correlation studies, respectively.

If we use the reported data of Evans et al. (2018), the metric $\Delta Z_{\mathrm{TiO}-\mathrm{H}_{2} \mathrm{O}} / H_{\mathrm{eq}} \sim-0.17$ when we average between wavelengths $0.555 \mu \mathrm{m}-0.6721 \mu \mathrm{m}$ for $Z_{\text {TiO }}$. This result is consistent with the aforementioned works, which conclude that $\mathrm{TiO}$ is not detected in the atmosphere of this planet. This measurement could be 
consistent with a solar $\mathrm{C} / \mathrm{O}$ and super-solar $\mathrm{O} / \mathrm{H}(\sim 10 \times$ solar $)$; however, given that Merritt et al. (2020) report non-detections of both $\mathrm{TiO}$ and $\mathrm{VO}$, our method is questionable for such weak signals.

Even more recently, Hoeijmakers et al. (2020) completed a molecule and atomic survey of WASP-121b using highresolution spectroscopy. These latter authors similarly find no $\mathrm{TiO}$ or Ti suggesting that a cold trap could be responsible for a global depletion in titanium in the atmosphere. They predict that $\mathrm{TiO}_{2}$ could represent an important absorber in the atmosphere, but without an accurate line list its detection with high-resolution spectroscopy will be difficult. We include $\mathrm{TiO}_{2}$ in our chemical model and find that its abundance is - at most - roughly an order of magnitude less abundant than $\mathrm{TiO}$, and strongly depends on the bulk $\mathrm{O} / \mathrm{H}$. The role that $\mathrm{TiO}_{2}$ will play in probing the chemical structure of exoplanetary atmospheres remains to be seen.

\subsection{WASP-74b}

Mancini et al. (2019) studied the transmission spectra of WASP$74 \mathrm{~b}$ between $500 \mathrm{~nm}$ and $2150 \mathrm{~nm}$. These latter authors found that their best fit for their optical data was a solar $\mathrm{C} / \mathrm{O}$ with the inclusion of $\mathrm{TiO}$ and VO. However, a higher $\mathrm{C} / \mathrm{O}$ model $(=1.1)$ gives a better fit to their data at $\lambda \sim 1625 \mathrm{~nm}$ (see their Fig. 8). A higher $\mathrm{C} / \mathrm{O}$ would make their measurement of $\Delta Z_{\mathrm{TiO}-\mathrm{H}_{2} \mathrm{O}} / H_{\text {eq }}$ (square point in Fig. 11) more consistent with our results, since a horizontal shift to $\mathrm{C} / \mathrm{O} \sim 0.8$ would make their estimation agree with our models - as we assumed a solar $\mathrm{O} / \mathrm{H}$.

The wavelength range around $1600 \mathrm{~nm}$ has also been suggested as a region to search for nitrogen-bearing species like $\mathrm{NH}_{3}$ and HCN by MacDonald \& Madhusudhan (2017). As such, a possible explanation for the discrepancy seen in our models with respect to the measurement of Mancini et al. (2019) is a high bulk nitrogen abundance in its atmosphere. If the oxygen abundance is reduced by a factor of a few, then the combination of a supersolar $\mathrm{C} / \mathrm{O}$ and reduced $\mathrm{O} / \mathrm{H}$ would make their measurement more consistent with our models.

\subsection{WASP-19b}

Sedaghati et al. (2017) studied the optical transmission spectra of WAPS-19b and suggested that many features were due to the existence of TiO. This conclusion was in contrast with the work of Huitson et al. (2013), who detected water absorption in the atmosphere of WASP-19b and therefore did not expect a high $\mathrm{C} / \mathrm{O}$ ratio and suggested an absence of TiO. Meanwhile, Madhusudhan (2012) favours a high $\mathrm{C} / \mathrm{O}(\geq 1)$ for this planet based on ground-based and Spitzer photometric data (we set $\mathrm{C} / \mathrm{O}=1$ in Fig. 11). We used the TiO detection of Sedaghati et al. (2017) and the water detection near $1.4 \mu \mathrm{m}$ of Huitson et al. (2013) to compute the metric for Fig. 11.

We find that the measurement of $\Delta Z_{\mathrm{TiO}_{-} \mathrm{H}_{2} \mathrm{O}} / H_{\mathrm{eq}}$ for WASP$19 \mathrm{~b}$ falls under the results of our chemical model. One way to overcome the discrepancy between our models and the observational work, as well as to explain the observations of both Huitson et al. (2013) and Sedaghati et al. (2017), would be to consider an enhanced oxygen abundance for the planet with respect to the solar value of $\mathrm{O} / \mathrm{H}$. In this way, the diamond point on Fig. 11 would shift to the left and the colour of the point would become darker (since $\mathrm{C} / \mathrm{O}$ would be lower and $\mathrm{O} / \mathrm{H}$ would be higher). The increased $\mathrm{O} / \mathrm{H}$ would likely produce a stronger $\mathrm{H}_{2} \mathrm{O}$ signal, which might explain the discrepancy between model and observations (as in Huitson et al. 2013; their bottom left panel of Fig. 19). An oxygen enhancement of 2 would help to improve the comparison between observations and our chemical models.

We note that Espinoza et al. (2019) additionally studied the optical transmission spectra of WASP-19b using the Magellan/IMACS spectrograph. These latter authors report an almost featureless transmission spectrum in their $0.45-0.9 \mu \mathrm{m}$ window - precisely where we would expect to see features from TiO. Espinoza et al. (2019) hypothesise that the TiO signal that is reported by Sedaghati et al. (2017) was actually due to starspots or faculae. Of the multiple transits reported by these latter authors, only one showed convincing evidence of $\mathrm{TiO}$ features. This transit also had the strongest evidence of stellar contamination, casting doubt on the detection. Clearly more observations of this planet are needed to conclusively constrain the presence (or absence) of $\mathrm{TiO}$.

\section{4. $H D 209458 b$}

The TiO abundance was inferred by Désert et al. (2008), and we use the $\mathrm{C} / \mathrm{O}$ and $\mathrm{O} / \mathrm{H}$ as inferred by Hobbs et al. (2019). Hobbs et al. (2019) inferred a high $\mathrm{C} / \mathrm{O}$ (between 0.9 and 1.0), assuming solar $\mathrm{O} / \mathrm{H}$ (they suggest $\mathrm{N} / \mathrm{O} \geq 1$ to explain the detection of $\mathrm{HCN}$ in the atmosphere). According to our chemical models, these ratios are inconsistent with the observed strength in the TiO transmission signal. Indeed, Désert et al. (2008) struggled to find a signature of $\mathrm{TiO}$, and needed to subtract the signal from sodium and potassium to find evidence for TiO.

The weak $\mathrm{TiO}$ signal is attributed to an abundance of $\mathrm{TiO}$ that is lower by a factor of between $10^{-4}$ and $10^{-3}$ than the solar abundance of titanium. Such a reduction could be contributed to the condensation of the molecule into clouds, followed by it raining out of the upper atmosphere. Since HD 209458b is cooler than the ultra-hot models we consider here (we used an ultrahot version of HD 209458b), then it is reasonable to assume that cloud formation could be greatly impacting our results. In fact, three-dimensional global circulation models show that cold traps on the night side of the planet can deplete $\mathrm{TiO}$ from the upper atmosphere if it condenses into sufficently large particle size (Parmentier et al. 2013). Accurate treatment of the chemical kinetics and cloud formation would be a useful avenue to further our study of this planetary atmosphere; however, for now, all we can say is that non-equilibrium (gas phase) chemistry and $\mathrm{TiO}$ formation is insufficient to explain the detection of $\mathrm{TiO}$.

\subsection{WASP-76b}

$\mathrm{Fu}$ et al. (2020) studied the optical and infrared transmission spectrum of WASP-76b between 0.3 and $1.5 \mu \mathrm{m}$ with HST STIS and WFC3, as well as the emission spectrum from between 1.2 and $1.6 \mu \mathrm{m}$, along with Spitzer data. We used their reported transmission spectrum data near the $0.6 \mu \mathrm{m}$ feature of $\mathrm{TiO}$ and around the $1.4 \mu \mathrm{m}$ feature of $\mathrm{H}_{2} \mathrm{O}$ to compute the metric for Fig. 11. Their fitting suggested a slightly super-solar $\mathrm{C} / \mathrm{O}$ (but matching solar within errors); their preferred $\mathrm{O} / \mathrm{H}$ is not well constrained, but is marginally subsolar. Our chemical model actually suggests a super-solar oxygen abundance to explain the metric in Fig. 11.

von Essen et al. (2020) and Edwards et al. (2020) performed similar studies of the atmosphere of WASP-76b with HST. Edwards et al. (2020) studied both transmission and emission spectra from 1.1 to $1.6 \mu \mathrm{m}$, and found evidence for both $\mathrm{TiO}$ and $\mathrm{H}_{2} \mathrm{O}$ in their fits, albeit with higher $\mathrm{TiO}$ abundance (by a factor of 1000) in their emission spectra than in their transmission spectra. Due to their choice of wavelength range, 
we did not include their data in our analysis of WASP-76b. von Essen et al. (2020) studied the transmission spectra between 0.3 and $0.9 \mu \mathrm{m}$ and found no evidence for TiO; instead, they found evidence for $\mathrm{TiH}$ which has transitions over a similar wavelength range (this molecule is discussed in Sect. 6.1). Because these latter authors did not include data from the $1.4 \mu \mathrm{m}$ feature of water, we similarly did not include their data in our analysis.

It is curious that the three aforementioned papers result in such different conclusions regarding the abundance of TiO. Some of these discrepancies likely arise from the wavelength range of study, and the fact that emission and transmission spectra study different pressure regions in the atmosphere. Indeed, the work of von Essen et al. (2020) suggests a TiO abundance gradient through the atmosphere (as predicted by our chemical model), and the possible presence of $\mathrm{TiH}$ found by von Essen et al. (2020) suggests that more chemical modelling is required (discussed below).

\section{Discussion: can TiO be used to constrain elemental ratios?}

Our primary question in this work is whether we can use $\mathrm{TiO}$ as an alternative tracer for the bulk chemical composition of (ultra) hot Jupiters. Detecting TiO has a number of benefits because of its significant number of electron transitions in optical wavelengths, making it more efficiently observable from the ground. This helps chemical characterisation studies because groundbased observatories are more accessible, and can offer much higher spectral resolution than space-based observatories. This study also requires a detection of $\mathrm{H}_{2} \mathrm{O}$ in NIR, which can be more difficult from the ground, but recently has been demonstrated with high-resolution spectroscopy and cross-correlation (see e.g. Alonso-Floriano et al. 2019).

From Fig. 11, we can see that a single measurement of $\Delta Z_{\mathrm{TiO}-\mathrm{H}_{2} \mathrm{O}} / H_{\text {eq }}$ can at least constrain a maximal $\mathrm{C} / \mathrm{O}$ if the metric is relatively high (i.e. higher than 5). Otherwise, we found that the metric increases slightly as a function of $\mathrm{C} / \mathrm{O}$ (up to 0.85 in our model), and hence a single measurement of the metric would struggle to constrain both $\mathrm{C} / \mathrm{O}$ and $\mathrm{O} / \mathrm{H}$ simultaneously. A measurement of a nitrogen- or carbon-bearing species would greatly improve our constraints on the bulk chemical properties of the atmosphere. One of these species, along with the measurement of the water feature that must be made to construct the metric, would give us a constraint on one of the elemental ratios, which when combined with the metric could be used to constrain the other ratio.

As previously mentioned, our model turned out to be insensitive to $\mathrm{N} / \mathrm{H}$, as variations in $\mathrm{N} / \mathrm{O}$ are done by varying $\mathrm{O} / \mathrm{H}$; and increasing $\mathrm{N} / \mathrm{H}$ did not result in any change in $\mathrm{TiO}$ abundance. We did not expect this to be the case as two of the three $\mathrm{TiO}$ oxidisers are nitrogen-bearing species. However, we found that increases of N/H typically resulted in increases of $\mathrm{N}_{2}$ abundance, which did not translate into more abundant oxidisers and subsequently TiO. Nevertheless, we stress that our metric is sensitive to $\mathrm{C} / \mathrm{H}$ and $\mathrm{O} / \mathrm{H}$ and hence can still help to understand the bulk chemical properties of hot Jupiter atmospheres.

While the metric from Eq. (5) is meant to be model independent, our assumption that $\mathrm{TiO}$ and $\mathrm{H}_{2} \mathrm{O}$ are the primary contributors to the spectrum near 0.6 and $1.4 \mu \mathrm{m}$ respectively comes from our atmospheric models. Since our model turned out to be insensitive to $\mathrm{N} / \mathrm{H}$, a coincident measurement of a nitrogen-bearing species would help to characterise both $\mathrm{C} / \mathrm{O}$ and N/O. This could be done using $\mathrm{HCN}$ in the case of high $\mathrm{C} / \mathrm{O}$

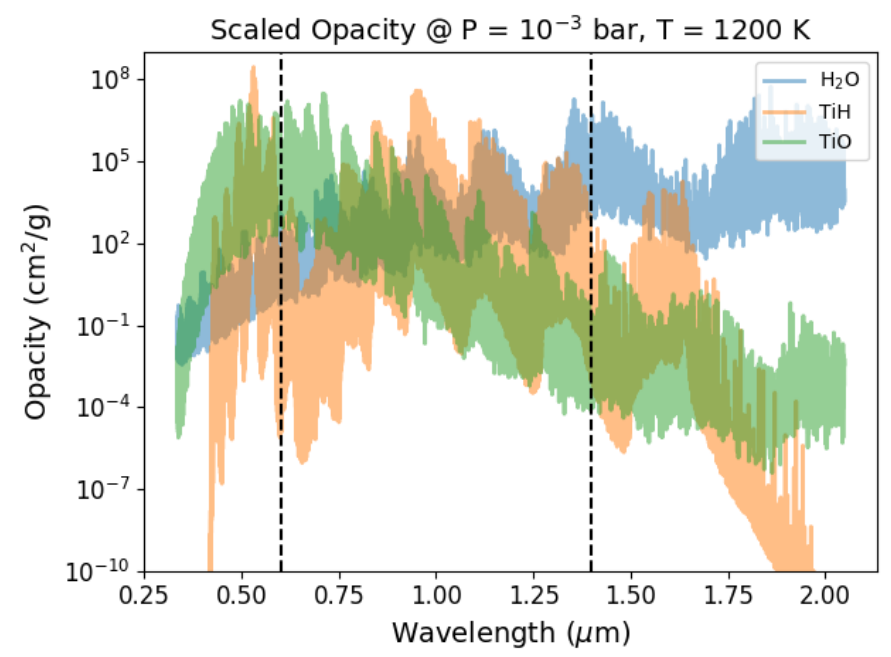

Fig. 12. Optical and NIR opacities for $\mathrm{TiO}, \mathrm{TiH}$, and $\mathrm{H}_{2} \mathrm{O}$. We scaled the opacity of $\mathrm{H}_{2} \mathrm{O}$ by a factor of $10^{4}$ to account for the larger abundance of $\mathrm{H}_{2} \mathrm{O}$ relative to titanium-bearing species in exoplanetary atmospheres. We note the two important wavelength ranges to the metric used in this paper with vertical dashed lines. Data taken from opacity.world on $12 / 03 / 2020$.

or with $\mathrm{NH}_{3}$ in the opposite case. There is already evidence of these molecules in hot Jupiter atmospheres (i.e. in MacDonald $\&$ Madhusudhan 2017) and they both happen to have features in the mid-IR which could be observable with JWST-MIRI. Nitrogen could prove to be a stronger tracer of planet formation as its chemistry is generally much simpler than carbon and oxygen. Indeed, analyses of the nitrogen abundance of Jupiter have already been used to place strong constraints on its formation history (Bosman et al. 2019; Öberg \& Wordsworth 2019).

\subsection{Incomplete titanium chemistry?}

In this work we focus primarily on the oxidation chemistry that leads to the generation of $\mathrm{TiO}$, further oxidation to $\mathrm{TiO}_{2}$, and the reverse reactions that would return titanium to its atomic state. Hot Jupiters are additionally hydrogen rich - recently new evidence has arisen for titanium monohydride (TiH) in the atmosphere of a hot Neptune (MacDonald \& Madhusudhan 2019), as well as in WASP-76b (von Essen et al. 2020) - and it is possible that chemical competition exists between $\mathrm{TiH}$ and $\mathrm{TiO}$. There is a known transition in $\mathrm{M}, \mathrm{L}$, and $\mathrm{T}$ dwarf stars where metal monohydrides appear in the atmospheres of the cooler $\mathrm{L}$ and $\mathrm{T}$ dwarfs at the expense of metal oxides, which are more abundant in warmer M dwarf stars (Kirkpatrick et al. 1999).

In an equilibrium chemistry calculation, Helling et al. (2019) showed that $\mathrm{TiH}$ tends to be underabundant relative to $\mathrm{TiO}$ for WASP-18b; however, as we discussed above, non-equilibrium chemistry can be important for the chemistry of TiO. Moving forward, a more complete chemical kinetic calculation of titanium chemistry should be done, including the production of $\mathrm{TiH}$. Because of the abundance of hydrogen in the atmospheres of giant planets, $\mathrm{TiH}$ could efficiently compete with $\mathrm{TiO}$ for the available titanium. TiH also has strong absorption bands in the optical, and could be confused in the transmission spectra of hot Jupiter atmospheres.

In Fig. 12 we illustrate this last point by plotting the opacities of $\mathrm{H}_{2} \mathrm{O}$, TiO, and $\mathrm{TiH}$. We scaled the opacity of $\mathrm{H}_{2} \mathrm{O}$ by a factor of $10^{4}$ to account for the fact that in atmospheres $\mathrm{H}_{2} \mathrm{O}$ is about four orders of magnitude more abundant than titanium-bearing 
molecules. We see near the wavelength range relevant to our metric that $\mathrm{TiH}$ has a feature that is as strong as that of $\mathrm{TiO}$, but is much less broad. At low spectral resolution and sensitivity it is possible that $\mathrm{TiO}$ features could be mistaken for $\mathrm{TiH}$ features.

\subsection{Are ultra-hot Jupiters the only place to look for TiO?}

We have intentionally focused on the ultra-hot population of hot Jupiters because they typically show the best evidence of TiO. The exception of this is HD 209458b, which shows weak evidence of TiO, but is not classified as an ultra-hot Jupiter. It is likely that high $(\geq 2000 \mathrm{~K})$ temperatures are needed for strong detections of $\mathrm{TiO}$ in the gas phase, since titanium-containing condensates are expected to be formed for temperatures below 2000 K (Lodders 2002).

While there are currently few planets that are classified as ultra-hot Jupiters ${ }^{8}$, the method outlined here can be very useful for characterising their chemical structure. There is still much to learn about the formation and orbital migration of these ultrahot Jupiters - and knowing from where they originate will be an important step in understanding their formation history.

Smaller and cooler planets will need other methods of tracing their chemical composition, since we do not expect to find high abundances of $\mathrm{TiO}$ in their atmospheres. Metal hydrides may be an interesting avenue of exploration because of their known connection to the lower temperature atmosphere of $\mathrm{L}$ and $\mathrm{T}$ dwarfs. Surveys of hot Jupiters with high-resolution spectroscopy from the ground would be a useful tool for studying atmospheric metal hydrides. Nevertheless, we will need a strong modelling effort to interpret their results. Ideally, the exoplanet community can draw from the knowledge already developed for studying dwarf stars ${ }^{9}$; however, non-equilibrium processes should be considered as hot Jupiters tend to have a bright UV source in their vicinity.

\section{Conclusions}

In this work we study the connection between detections of $\mathrm{TiO}$ and the bulk chemical composition of the atmospheres of hot Jupiters. We model non-equilibrium chemistry in the atmospheres of two ultra-hot Jupiter models while varying $\mathrm{C} / \mathrm{O}$ and $\mathrm{O} / \mathrm{H}$ using the astrochemical code VULCAN. We account for changes in the temperature and pressure structure of these atmospheres due to changes in $\mathrm{C} / \mathrm{O}$ by considering different structures for low $(<0.8)$, medium $(<0.95)$, and high $(=1) \mathrm{C} / \mathrm{O}$. We calculate synthetic spectra for our atmospheric models and compare them with observed transmission spectra that feature $\mathrm{TiO}$ detections.

We use the metric: $\Delta Z_{\mathrm{TiO}-\mathrm{H}_{2} \mathrm{O}} / H_{\text {eq }}$ to quantify our comparisons between model and observations. This metric should be independent of the physical properties of the planet, and should only depend on the abundance of a given molecule along the line of sight. With this metric we find that:

- WASP-121b is in good agreement with solar values of both $\mathrm{C} / \mathrm{O}$ and $\mathrm{O} / \mathrm{H}$.

- WASP-74b is more consistent with a super-solar C/O ( 0.8) for solar $\mathrm{O} / \mathrm{H}$, which partly agrees with the fit done by Mancini et al. (2019). Alternatively, if C/O is solar (as suggested by Mancini et al. 2019), then a reduction of $\mathrm{O} / \mathrm{H}$ by a factor of a few would make the measured strength of the $\mathrm{TiO}$ feature consistent with our chemical models.

8 On the NASA exoplanet archive there are currently $\sim 40$ planets with $T_{\text {eq }}>2000 \mathrm{~K}$.

9 Which typically rely on equilibrium chemistry.
- For WASP-19b, an enhancement in the oxygen abundance by a factor of two relative to solar would best explain the measured strength in the $\mathrm{TiO}$ feature. This enhancement relative to the reported values of $\mathrm{C} / \mathrm{O}$ would also help to explain the discrepancy between the studies of Huitson et al. (2013) and Sedaghati et al. (2017).

- HD 209458b shows only marginal TiO detections which could be caused by $\mathrm{TiO}$ condensation and cloud formation. These processes are not included in our chemical analysis, which makes an analysis of HD 209458b difficult in our framework.

- WASP-76b would require a higher oxygen abundance than is reported in observational papers to agree with our chemical model. We require super-solar $\mathrm{O} / \mathrm{H}$ while observational fits prefer subsolar $\mathrm{O} / \mathrm{H}$.

We find that $\mathrm{TiO}$ detections are a possible tracer of the bulk composition; however, we may be limited to ultra-hot Jupiters with effective temperatures $>2000 \mathrm{~K}$. For complete characterisations, a detection of a nitrogen or carbon carrier is needed to pin down both $\mathrm{C} / \mathrm{O}$ and $\mathrm{O} / \mathrm{H}$. Since our method turned out to not depend on $\mathrm{N} / \mathrm{H}$ (but rather on $\mathrm{O} / \mathrm{H}$ by construction) the detection of a nitrogen carrying molecule - like $\mathrm{HCN}$ and $\mathrm{NH}_{3}-$ would be required to give us enough data to constrain the atmospheric N/O, which currently stands as a complete unknown for exoplanetary systems.

With new missions like the James Webb Space Telescope coming online in the near future, exoplanetary science will have a never-before-seen view of exoplanetary atmospheres in the mid-IR. This will undoubtedly revolutionise our understanding of the bulk chemical composition of exoplanetary atmospheres. However, studies like ours show that efforts in the optical can still provide a unique view of the problem, and, furthermore, alternative tracers of bulk chemical composition should be explored. We are confident that the findings of this work will contribute to this task. Furthermore, astrochemical modelling will continue to help plan, search, detect, and characterise more exoplanetary atmospheres as the data become available.

Acknowledgements. We thank the anonymous referee for their helpful comments that improved the clarity of this manuscript. V.R. is supported by Leiden Observatory through the Oort Scholarship, and was funded as part of the Leiden/ESA Astrophysics Program for Summer students (LEAPS) in the summer of 2019. A.J.C acknowledges support by the European Union ERC grant H2020 ExoplanetBio supervised by Ignas Snellen. P.M. acknowledges support from the European Research Council under the European Union's Horizon 2020 research and innovation program under grant agreement No. 832428. Astrochemistry in Leiden is supported by the European Union A-ERC grant 291141 CHEMPLAN, by the Netherlands Research School for Astronomy (NOVA), and by a Royal Netherlands Academy of Arts and Sciences (KNAW) professor prize.

\section{References}

Alonso-Floriano, F. J., Sánchez-López, A., Snellen, I. A. G., et al. 2019, A\&A, 621, A74

Asplund, M., Grevesse, N., Sauval, A. J., \& Scott, P. 2009, ARA\&A, 47, 481

Banks, P. M., \& Kockarts, G. 1973, Aeronomy (Amsterdam: Elsevier)

Benneke, B. 2015, ArXiv e-prints [arXiv:1504.07655]

Birkby, J. L., de Kok, R. J., Brogi, M., Schwarz, H., \& Snellen, I. A. G. 2017, AJ, 153,138

Blecic, J., Harrington, J., \& Bowman, M. O. 2016, ApJS, 225, 4

Bosman, A. D., Cridland, A. J., \& Miguel, Y. 2019, A\&A, 632, L11

Brewer, J. M., \& Fischer, D. A. 2016, ApJ, 831, 20

Brogi, M., Snellen, I. A. G., de Kok, R. J., et al. 2013, ApJ, 767, 27

Cridland, A. J., Pudritz, R. E., \& Alessi, M. 2016, MNRAS, 461, 3274

Cridland, A. J., Pudritz, R. E., Birnstiel, T., Cleeves, L. I., \& Bergin, E. A. 2017, MNRAS, 469, 3910

Cridland, A. J., van Dishoeck, E. F., Alessi, M., \& Pudritz, R. E. 2019a, A\&A, 632, A63 
Cridland, A. J., Eistrup, C., \& van Dishoeck, E. F. 2019b, A\&A, 627, A127

Désert, J. M., Vidal-Madjar, A., Lecavelier Des Etangs, A., et al. 2008, A\&A, 492,585

Dhital, S., West, A. A., Stassun, K. G., et al. 2012, AJ, 143, 67

Edwards, B., Changeat, Q., Baeyens, R., et al. 2020, AJ, 160, 8

Eistrup, C., Walsh, C., \& van Dishoeck, E. F. 2018, A\&A, 613, A14

Espinoza, N., Rackham, B. V., Jordán, A., et al. 2019, MNRAS, 482, 2065

Evans, T. M., Sing, D. K., Wakeford, H. R., et al. 2016, ApJ, 822, L4

Evans, T. M., Sing, D. K., Goyal, J. M., et al. 2018, AJ, 156, 283

Fu, G., Deming, D., Lothringer, J., et al. 2020, ArXiv e-prints [arXiv:2005.02568]

Gandhi, S., \& Madhusudhan, N. 2019, MNRAS, 485, 5817

Gravity Collaboration (Nowak, M., et al.) 2020, A\&A, 633, A110

Helling, C., Woitke, P., Rimmer, P. B., et al. 2014, Life, 4, 142

Helling, C., Gourbin, P., Woitke, P., \& Parmentier, V. 2019, A\&A, 626, A133

Hobbs, R., Shorttle, O., Madhusudhan, N., \& Rimmer, P. 2019, MNRAS, 487, 2242

Hoeijmakers, H. J., Seidel, J. V., Pino, L., et al. 2020, ArXiv e-prints [arXiv:2006.11308]

Huitson, C. M., Sing, D. K., Pont, F., et al. 2013, MNRAS, 434, 3252

Kesseli, A. Y., Kirkpatrick, J. D., Fajardo-Acosta, S. B., et al. 2019, AJ, 157, 63

Kippenhahn, R., \& Weigert, A. 1990, Stellar Structure and Evolution (Berlin: Springer)

Kirkpatrick, J. D., Reid, I. N., Liebert, J., et al. 1999, ApJ, 519, 802

Kreidberg, L., Bean, J. L., Désert, J.-M., et al. 2014, ApJ, 793, L27

Lecavelier Des Etangs, A., Vidal-Madjar, A., Désert, J. M., \& Sing, D. 2008, A\&A, 485, 865

Lodders, K. 2002, ApJ, 577, 974

Lodders, K., Palme, H., \& Gail, H. P. 2009, Solar System, Landolt Börnstein, Group VI Astronomy and Astrophysics (Berlin: Springer), 4B, 712

Lothringer, J. D., Barman, T., \& Koskinen, T. 2018, ApJ, 866, 27

Lothringer, J. D., Fu, G., Sing, D. K., \& Barman, T. S. 2020, ApJ, 898, L14

MacDonald, R. J., \& Madhusudhan, N. 2017, MNRAS, 469, 1979

MacDonald, R. J., \& Madhusudhan, N. 2019, MNRAS, 486, 1292

Madhusudhan, N. 2012, ApJ, 758, 36

Madhusudhan, N., \& Seager, S. 2011, ApJ, 729, 41

Madhusudhan, N., Harrington, J., Stevenson, K. B., et al. 2011, Nature, 469, 64

Madhusudhan, N., Amin, M. A., \& Kennedy, G. M. 2014, ApJ, 794, L12

Mancini, L., Southworth, J., Mollière, P., et al. 2019, MNRAS, 485, 5168

Merritt, S. R., Gibson, N. P., Nugroho, S. K., et al. 2020, A\&A, 636, A117

Mollière, P., van Boekel, R., Dullemond, C., Henning, T., \& Mordasini, C. 2015, ApJ, 813, 47

Mollière, P., van Boekel, R., Bouwman, J., et al. 2017, A\&A, 600, A10

Mollière, P., Wardenier, J. P., van Boekel, R., et al. 2019, A\&A, 627, A67

Mordasini, C., Klahr, H., Alibert, Y., Miller, N., \& Henning, T. 2014, A\&A, 566, A141

Mordasini, C., van Boekel, R., Mollière, P., Henning, T., \& Benneke, B. 2016 ApJ, 832, 41

Morley, C. V., Knutson, H., Line, M., et al. 2017, AJ, 153, 86

Moses, J. I., Visscher, C., Fortney, J. J., et al. 2011, ApJ, 737, 15

Öberg, K. I., \& Wordsworth, R. 2019, AJ, 158, 194

Öberg, K. I., Murray-Clay, R., \& Bergin, E. A. 2011, ApJ, 743, L16

Oberg, K., Bergin, E., Andrews, S., et al. 2019, BAAS, 51, 165

Parmentier, V., Showman, A. P., \& Lian, Y. 2013, A\&A, 558, A91

Pudritz, R. E., Cridland, A. J., \& Alessi, M. 2018, Handbook of Exoplanets (Berlin: Springer), 144

Ritter, D., \& Weisshaar, J. C. 1989, J. Phys. Chem., 93, 1576

Ritter, D., \& Weisshaar, J. C. 1990, J. Phys. Chem., 94, 4907

Sedaghati, E., Boffin, H. M. J., MacDonald, R. J., et al. 2017, Nature, 549, 238

Sing, D. K., Wakeford, H. R., Showman, A. P., et al. 2015, MNRAS, 446, 2428
Stevenson, K. B., Bean, J. L., Seifahrt, A., et al. 2014, AJ, 147, 161 Tsai, S.-M., Lyons, J. R., Grosheintz, L., et al. 2017, ApJS, 228, 20

Veyette, M. J., Muirhead, P. S., Mann, A. W., \& Allard, F. 2016, ApJ, 828, 95 von Essen, C., Mallonn, M., Hermansen, S., et al. 2020, A\&A, 637, A76

\section{Appendix A: Variations in $\mathrm{N} / \mathrm{O}$ caused by changing $\mathrm{N} / \mathrm{H}$}

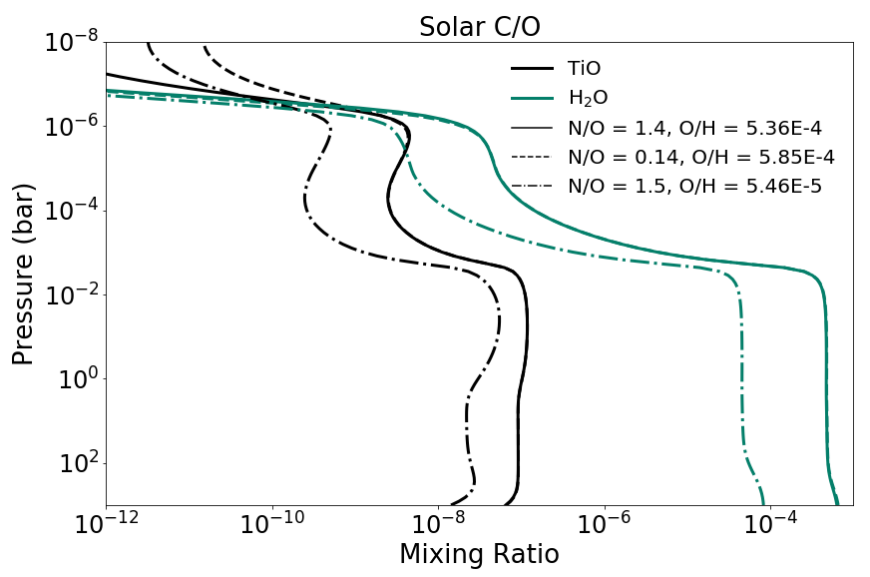

Fig. A.1. Variation in water and $\mathrm{TiO}$ abundances caused by variations in bulk N/O for solar C/O. Here we varied N/O by increasing N/H by an order of magnitude and compared the findings with those of the models in the main text which kept $\mathrm{N} / \mathrm{H}$ constant.

On the onset of this work, we assumed that N/O would impact the abundance of $\mathrm{TiO}$ because of the resulting abundance of the molecules responsible for the oxidation of atomic Ti. Our method involved varying $\mathrm{N} / \mathrm{O}$ by changing $\mathrm{O} / \mathrm{H}$ while keeping $\mathrm{N} / \mathrm{H}$ constant. Here we check whether variations in $\mathrm{N} / \mathrm{H}$ (for constant $\mathrm{O} / \mathrm{H}$ ) have the same effect on the abundance of $\mathrm{TiO}$.

In Fig. A.1, we show the result of increasing N/H by an order of magnitude and comparing its results to two other models used in the main text. We find that increasing $\mathrm{N} / \mathrm{H}$ by an order of magnitude does not have the equivalent effect on the resulting abundance of $\mathrm{TiO}$ as decreasing $\mathrm{O} / \mathrm{H}$ by an order of magnitude (even though their N/O are the same - see the solid and dot-dashed lines).

The difference in increasing $\mathrm{N} / \mathrm{H}$ and decreasing $\mathrm{O} / \mathrm{H}$ can be easily explained by considering the primary nitrogen carrier: $\mathrm{N}_{2}$. Increasing $\mathrm{N} / \mathrm{H}$ by an order of magnitude generally results in an increase in the $\mathrm{N}_{2}$ abundance, but has a much smaller effect on the $\mathrm{TiO}$ oxidisers. An increase in $\mathrm{N} / \mathrm{H}$ does not strictly translate to an increase in $\mathrm{NO}$ abundance (for example) because there is no more available oxygen for its production. However, when $\mathrm{O} / \mathrm{H}$ is decreased, the abundance of NO will also see a decrease in its abundance (and subsequently $\mathrm{TiO}$ ). 